\title{
Gamma-ray Bursts Cosmology with The X-ray Fundamental Plane Relation
}

Maria Dainotti ( $\square$ mariagiovannadainotti@yahoo.it)

Jagellonian University

Jacob Fernandez

University of Santa Barbara

Giuseppe Saraccino

University of Naples Federico II

Aleksander Lenart

Jagiellonian University

Sergey Postnikov

DAVER International Inc., CEO, Windsor, ON N9E 2 G8

Shigehiro Nagataki

RIKEN

Nissim Fraija

Universidad Nacional Autonoma de Mexico

Physical Sciences - Article

Keywords: Gamma-ray Bursts Cosmology, X-ray Fundamental Plane Relation

Posted Date: November 30th, 2020

DOI: https://doi.org/10.21203/rs.3.rs-108982/v1

License: (c) (1) This work is licensed under a Creative Commons Attribution 4.0 International License.

Read Full License 


\section{Gamma-ray Bursts Cosmology with The X-ray Fundamen- tal Plane Relation}

Dainotti, M. G. ${ }^{1,2,3,4,5}$, Fernandez, J. ${ }^{6}$, Sarracino, G. ${ }^{8,9}$, Lenart, A. Ł. ${ }^{5}$, Postnikov S. ${ }^{7}$, Nagataki, S. ${ }^{1}$, Fraija, N. ${ }^{10}$

${ }^{1}$ Interdisciplinary Theoretical \& Mathematical Science Program, RIKEN (iTHEMS), 2-1 Hirosawa, Wako, Saitama, Japan 351-0198;

${ }^{2}$ SLAC NATIONAL ACCELERATOR LABORATORY 2575 Sand Hill Road, Menlo Park, CA 94025, USA;

${ }^{3}$ Department of Physics \& Astronomy, Stanford University, Via Pueblo Mall 382, Stanford, CA 94305-4060, USA;

${ }^{4}$ Space Science Institute, Boulder, Colorado

${ }^{5}$ Astronomical Observatory, Jagiellonian University, ul. Orla 171, 31-501 Kraków, Poland;

${ }^{6}$ Department of Physics, University of California, Santa Barbara, CA 93106;

${ }^{7}$ DAVER International Inc., CEO, Windsor, ON N9E 2G8, Canada;

${ }^{8}$ Dipartimento di Fisica, "E. Pancini” Università “Federico II” di Napoli, Compl. Univ. Monte S. Angelo Ed. G, Via Cinthia, I-80126 Napoli (Italy)

${ }^{9}$ INFN Sez. di Napoli, Compl. Univ. Monte S. Angelo Ed. G, Via Cinthia, I-80126 Napoli (Italy)

${ }^{10}$ Instituto de Astronomía, Universidad Nacional Autónoma de México, Apdo. Postal 70-264, Cd. Universitaria, Ciudad de México 04510

Cosmological models and the value of their parameters are at the center of the debate be- 
cause of the tension between the results obtained by the SNe Ia data and the Plank ones of the Cosmic Microwave Background Radiation. Thus, adding cosmological probes observed at high redshifts, such as Gamma-Ray Bursts (GRBs), is needed. Using GRB correlations between luminosities and a cosmological independent variable is challenging because GRB luminosities vary widely. We corrected a tight correlation between the rest-frame end time of the X-ray plateau, its corresponding X-ray luminosity, and the peak prompt luminosity: the so-called fundamental plane relation, using the jet opening angle. Its intrinsic scatter is $0.017 \pm 0.010 \mathrm{dex}, 95 \%$ smaller than the isotropic fundamental plane relation, the smallest compared to any current GRB correlation in the literature. This shows that GRBs can be used as reliable cosmological tools. We use this GRB corrected correlation for the so-called platinum sample (a well-defined set with relatively flat plateaus), together with SNe Ia data, to constrain different cosmological parameters like the matter content of the universe today, $\Omega_{M}$, the Hubble constant $H_{0}$, and the dark energy parameter $w$ for a $w$ CDM model. We confirm the $\Lambda$ CDM model but using GRBs up to $z=5$, a redshift range much larger than one of SNe Ia.

Gamma-Ray Bursts (GRBs) are the most luminous panchromatic transient phenomena in the universe after the Big Bang, and are among the farthest astrophysical objects ever observed. One of the most challenging goals in modern astrophysics is their use as standard candles. GRBs' potential use as cosmological tools is similar to what has been achieved for SNe Ia, but GRBs are observed out to much larger redshift $(z=9.4)$, which allows us to extend the cosmological ladder at much larger distances than SNe Ia, observed up to $z=2.3^{1}$. However, to use GRBs as cosmological 
tools, we need to understand their emission mechanisms. Indeed, there is still an ongoing debate regarding their physical mechanisms and their progenitors. There are several proposed scenarios regarding their possible origin: e.g., the explosions of extremely massive stars and the merging of two compact objects, like neutron stars (NSs) and black holes (BHs). Both these models consider ordinary NSs, BHs or fast spinning newly born highly magnetized NSs (magnetars) as the central engines of the GRBs' energy emission. In the former scenario, the compact object acting as the central engine is the remnant of the massive star after its collapse, while the latter results from the coalescence of the two compact objects and its subsequent explosion.

To pinpoint the different possible origins, we need to categorize GRBs according to their phenomenology. The GRB prompt emission is usually observed from hard X-rays to $\geq 100$ $\mathrm{MeV} \gamma$-rays, and sometimes also in optical wavelengths. The afterglow is the long-lasting multiwavelength emission (in X-rays, optical, and sometimes radio) following the prompt.

GRBs are traditionally classified as short and long GRBs, depending on the prompt emission duration: $T_{90} \leq 2 \mathrm{~s}$ or $T_{90} \geq 2 \mathrm{~s}^{1}$, respectively ${ }^{2,3}$.

The Neil Gehrels Swift Observatory (hereafter Swift) records the observations of the X-ray plateau emission ${ }^{4-6}$ which generally lasts from $10^{2}$ to $10^{5} \mathrm{~s}$ and is followed by a power law (PL) decay phase. Several models have been proposed to explain the plateau: the long-lasting energy injection into the external shock, where a single relativistic blast wave interacts with the surrounding medium ${ }^{7}$ or the spin-down luminosity of a newly born magnetar ${ }^{8}$. Several correlations involving

\footnotetext{
${ }^{1} T_{90}$ is the time over which a burst emits from $5 \%$ to $95 \%$ of its total measured counts in the prompt emission.
} 
the plateau ${ }^{9-13}$ and their applications as cosmological tools have been discussed in the literature so far $^{14-16}$. One remarkable correlation which involves the plateau emission is the so-called Dainotti relation, which links the rest frame time at the end of the plateau emission, $T_{X}^{*}$, with its correspondent luminosity, $L_{X}{ }^{10}$. This correlation can be explained naturally within the magnetar scenario ${ }^{8,17}$, and it indicates that the energy reservoir of the plateau is constant. An extension of this correlation in three dimensions has been discovered by adding the prompt emission's peak luminosity, $L_{\text {peak }}{ }^{9,18}$. The Dainotti 3D relation, the so-called fundamental plane correlation, defines a plane whose axes are $L_{X}, T_{X}^{*}$ and $L_{\text {peak }}$.

To boost the use of this correlation as a cosmological tool, as pointed out in ${ }^{9,10,18}$, we need to select a subsample of GRBs with very well-defined properties from both a morphological and a physical point of view. We focus our attention on a sample with well-defined and almost flat plateaus, called the platinum sample, whose features are detailed in Methods.

However, the mentioned relations and many others do not consider the collimated nature of GRBs, which leads to an overestimation of the luminosities and the energies. The collimated nature of GRBs is widely accepted from a theoretical point of view: their emission is beamed into a jet, enclosed into the so-called jet opening angle $\left(\theta_{j e t}\right)^{19}$. This fact requires the isotropic luminosities, $L_{i s o}$, to be corrected in the following way: $L_{j e t}=L_{i s o}\left(1-\cos \left(\theta_{j e t}\right)\right)$, which can decrease the inferred luminosities associated with GRBs by two or three orders of magnitude concerning a more simplified isotropic assumption. Thus, we here use the tightest three-parameter correlation in the literature, which is the fundamental plane relation introduced before, and we correct it for $\theta_{j e t}$, see 
Methods. First, we have selected 214 GRB X-ray plateau afterglows detected by Swift from 2005 January up to 2019 August with known redshifts, spectroscopic or photometric, available in ${ }^{20}$, on the Greiner web page ${ }^{2}$ and in the Gamma-ray Coordinates Network (GCN) circulars and notices ${ }^{3}$, excluding redshifts for which there is only a lower or an upper limit. We include all GRBs for which the Burst Alert Telescope (BAT) and X-Ray Telescope (XRT) light curves can be fitted by the phenomenological ${ }^{21}$ model, see Methods. We then choose from the whole sample a subset of long GRBs with very well-defined selection criteria, the so-called platinum sample. Fig. 1 shows the isotropic fundamental plane correlation for the platinum sample in 3D (upper panel), and the same correlation corrected by $\theta_{j e t}$ (lower panel). We see a great reduction of the intrinsic scatter, $\sigma_{\text {int }}(95 \%)$, from the isotropic to the jetted fundamental plane.

The isotropic fundamental plane relation can be written in the following way:

$$
\log L_{X}=C_{\text {iso }}+a_{\text {iso }} \times \log T_{X}^{*}+b_{\text {iso }} \times\left(\log L_{\text {peak }}\right)
$$

where $a_{i s o}$ and $b_{i s o}$ are the best fit parameters given by the D'Agostini ${ }^{22}$ fitting related to $\log T_{X}^{*}$ and $\log L_{\text {peak }}$, respectively, while $C_{i s o}$ is the normalization. The best fits are $a_{i s o}=-0.87 \pm 0.11$, $b_{\text {iso }}=0.54 \pm 0.07, C_{\text {iso }}=22.95 \pm 3.85$, and $\sigma_{\text {int }}=0.34 \pm 0.04$.

The fundamental plane relation with the correction introduced by $\theta_{j e t}$ can be written as follows:

$$
\log L_{X}+\log \left(1-\cos \left(\theta_{j e t}\right)\right)=C_{j e t}+a_{j e t} \times \log T_{X}^{*}+b_{j e t} \times\left(\log L_{\text {peak }}+\log \left(1-\cos \left(\theta_{j e t}\right)\right)\right)
$$

where $a_{j e t}$ and $b_{j e t}$ are the best fit parameters given by the D'Agostini fitting related to $\log T_{X}^{*}$ and

\footnotetext{
${ }^{2}$ https://www.mpe.mpg.de/ jcg/grbgen.html

${ }^{3}$ http://gcn.gsfc.nasa.gov/
} 
$\log L_{\text {peak }}$, respectively, while $C_{\text {jet }}$ is the normalization. The best fits are $a_{\text {jet }}=-0.93 \pm 0.03$, $b_{\text {jet }}=0.25 \pm 0.03, C_{\text {jet }}=36.70 \pm 1.50$, and $\sigma_{\text {int }, j e t}=0.017 \pm 0.010$. The contour plots of these parameters are shown in Fig. 4.

The jetted fundamental plane yields a reduction of $\sigma_{i n t}$ of $95 \%$ for the platinum sample with respect to the isotropic one. This value of $\sigma_{\text {int }}$ for this sample is the smallest in the literature regarding GRBs correlations so far. Furthermore, we have shown that this method reduces the intrinsic scatter to the extent where it is possible to estimate cosmological parameters, such as the matter content of the universe at today, $\Omega_{M}$, using this GRBs' correlation. The jetted fundamental plane shown in Eq. 2 can be expressed in terms of the X-ray flux at the end of the plateau, the luminosity distance, $D_{L}\left(z, \Omega_{M}, w, H_{0}\right)$, the peak prompt flux and the rest frame end time of plateau, $T_{X}^{*}$. In this fitting, we use this relation for the platinum sample together with the Pantheon sample of SNe Ia ${ }^{23}$. To overcome the so-called circularity problem, we here change contemporaneously the cosmological parameters together with the fitting coefficients of the fundamental plane. This method of obtaining the parameters is powerful, since it does not need any calibration of the fundamental plane relation on other local probes. Namely, the parameters of the correlations are not fixed but free to vary, following an approach already tested in ${ }^{16}$. One of our main results is shown in Fig. 2, where we have varied $H_{0}$ fixing $\Omega_{M}=0.3$ in the $\Lambda$ CDM model and we have obtained $H_{0}=69.97 \pm 0.13 \mathrm{Km} \mathrm{s}^{-1} \mathrm{Mpc}^{-1}$, which has a reduced scatter by $15.4 \%$ compared to the result obtained by using SNe Ia alone. We here stress that this reduction of the scatter is much larger than the increase in the percentage of the number of GRBs added to the SNe Ia (50 GRBs is just an increase of $4.8 \%$ compared to the Pantheon sample of 1048 SNe Ia). When we consider the result 
achieved by varying $\Omega_{M}$ and $H_{0}$ simultaneously with GRBs+SNe Ia we obtain $H_{0}=69.89 \pm 0.34$ $K m s^{-1} M p c^{-1}$, which allows a reduced $H_{0}$ tension at $2.96 \sigma$ level compared to the value of $H_{0}$ obtained with Planck measurements, $H_{0}=67.4 \pm 0.5 \mathrm{Km} \mathrm{s}^{-1} \mathrm{Mpc}^{-124}$. This result shows that the $H_{0}$ tension is comparable with the one obtained with only the Pantheon sample, where the tension is at $3.07 \sigma$ level. Another significant result is the reduction of the error on $\Omega_{M}$ of $11 \%$ when we use GRBs+SNe Ia together, and we fix the value of $H_{0}=70 \mathrm{Km} \mathrm{s}^{-1} \mathrm{Mpc}^{-1}$ at a fiducial value with respect of the result achieved using only SNe Ia data. Indeed, We have obtained $\Omega_{M}=0.299 \pm 0.009$ with GRBs+SNe Ia versus $\Omega_{M}=0.298 \pm 0.010$ obtained with SNe Ia only. These results are striking because our computed value of $\Omega_{M}$ using the GRB fundamental plane relation corrected for $\theta_{j e t}$ together with SNe Ia data is consistent with the one provided in the literature within the $\Lambda \mathrm{CDM}$ model $\left(\Omega_{M}=0.284 \pm 0.012\right.$ from SNe Ia data $\left.{ }^{23}\right)$ To further confirm the reliability of our conclusions and as a consistency check, we computed the same parameters using only SNe Ia. Results are summarized in Table 1.

We stress that these results are compatible with the $\Lambda$ CDM model. The great advantage is that we have computed them with high- $z$ probes up to $z=5$. The reduction of $\sigma_{\text {int }}$ after the correction introduced by $\theta_{\text {jet }}$ allows GRBs to be reliable standard candles, together with $\mathrm{SNe}$ Ia, to provide a more precise estimate for these cosmological parameters.

\section{References}




\begin{tabular}{|c|c|c|c|c|}
\hline SNe la sample & Model & $w$ & $\Omega_{M}$ & $H_{0}$ \\
\hline \hline (1) varying $\Omega_{M}$ & $\Lambda \mathrm{CDM}$ & $\mathbf{- 1}$ & $0.298 \pm 0.010$ & $\mathbf{7 0}$ \\
\hline (2) varying $H_{0}$ & $\Lambda \mathrm{CDM}$ & $\mathbf{- 1}$ & $\mathbf{0 . 3 0}$ & $69.96 \pm 0.15$ \\
\hline (3) varying $\Omega_{M}$ and $H_{0}$ & $\Lambda \mathrm{CDM}$ & $\mathbf{- 1}$ & $0.300 \pm 0.020$ & $69.97 \pm 0.33$ \\
\hline (4) varying $w$ & $w \mathrm{CDM}$ & $-1.006 \pm 0.019$ & $\mathbf{0 . 3 0}$ & $\mathbf{7 0}$ \\
\hline \hline SNe la + GRB sample & Model & $w$ & $\Omega_{M}$ & $H_{0}$ \\
\hline \hline (1) varying $\Omega_{M}$ & $\Lambda \mathrm{CDM}$ & $\mathbf{- 1}$ & $0.299 \pm 0.009$ & $\mathbf{7 0}$ \\
\hline (2) varying $H_{0}$ & $\Lambda \mathrm{CDM}$ & $\mathbf{- 1}$ & $\mathbf{0 . 3 0}$ & $69.97 \pm 0.13$ \\
\hline (3) varying $\Omega_{M}$ and $H_{0}$ & $\Lambda \mathrm{CDM}$ & $\mathbf{- 1}$ & $0.305 \pm 0.022$ & $69.89 \pm 0.34$ \\
\hline (4) varying $w$ & $w \mathrm{CDM}$ & $-1.006 \pm 0.019$ & $\mathbf{0 . 3 0}$ & $\mathbf{7 0}$ \\
\hline
\end{tabular}

Table 1: Results of the fitting of various cosmological parameters using the SNe la alone (the upper part of the table) and the GRB fundamental plane relation, corrected for the jet opening angle, using the platinum sample and the Pantheon sample for $\mathrm{SNe}$ la together (the lower part of the Table). The values in bold are the ones fixed in our computations at fiducial values. 
1. Rodney S. A., et al., Two SNe Ia at Redshift $\approx 2$ : Improved Classification and Redshift Determination with Medium-band Infrared Imaging, 2015, Astrophys. J., 150, 5;

2. Mazets, E. P., et al., Catalog of cosmic gamma-ray bursts from the KONUS experiment data, 1981, Astrophys. Space Sci., 80, 1, 3;

3. Kouveliotou, et al., Identification of Two Classes of Gamma-Ray Bursts, 1993, Astrophys. J., 413, 2, L101;

4. Evans, P. A., et al., Methods and results of an automatic analysis of a complete sample of Swift-XRT observations of GRBs, 2009, Mon. Not. R. Astron. Soc. , 397, 3, 1177

5. O’Brien, P. T., Willingale, R., et al., The Early X-Ray Emission from GRBs, 2006, Astrophys. $J ., 647,2,1213$;

6. Sakamoto, T., Hill J. E., et al., Evidence of Exponential Decay Emission in the Swift GammaRay Bursts, 2007, Astrophys. J., 669, 2, 1115;

7. Zhang, B., et al., Physical Processes Shaping Gamma-Ray Burst X-Ray Afterglow Light Curves: Theoretical Implications from the Swift X-Ray Telescope Observations, 2006, Astrophys. J., 642, 1, 354;

8. Stratta, G., Dainotti, M. G., Dall'Osso, S., Hernandez, X., De Cesare, G., On the magnetar origin of the GRBs presenting X-ray afterglow plateaus, 2018, Astrophys. J., 869, 2, 15;

9. Dainotti, M. G., Postnikov, S., Hernandez, X., Ostrowski, M., A Fundamental Plane for Long Gamma-Ray Bursts with X-Ray Plateaus, 2016, Astrophys. J. Let., 825, 2, id L20, 6 
10. Dainotti M. G., Cardone, V. F., Capozziello, S., A time luminosity correlation for GRBs in the X-rays, 2008, Mon. Not. R. Astron. Soc. Let., 391, 1;

11. Dainotti, M. G., Petrosian, V., Singal, J., Ostrowski, M., Slope evolution of GRB correlations and cosmology, 2013, Astrophys. J., 774, 157;

12. Dainotti, M. G., et al., Luminosity-time and luminosity-luminosity correlations for GRB prompt and afterglow plateau emissions, 2015, Mon. Not. R. Astron. Soc. , 451, 4;

13. Liang, E., et al., Constraining Gamma-ray Burst Initial Lorentz Factor with the Afterglow Onset Feature and Discovery of a Tight $\Gamma_{0}-E_{\gamma, \text { iso }}$ Correlation, 2010, Astrophys. J., 725, 2;

14. Cardone, V. F:, Dainotti, M. G., Capozziello, S., Willingale, R., Constraining cosmological parameters by gamma-ray burst X-ray afterglow light curves, 2010, Mon. Not. R. Astron. Soc. , 408, 2;

15. Postnikov, S., Dainotti, M. G., Hernandez, X., Capozziello, S., Nonparametric Study of the Evolution of the Cosmological Equation of State with SNeIa, BAO, and High-redshift GRBs, 2014, Astrophys. J., 783, 2, 126;

16. Dainotti, M. G., Cardone, V. F., Piedipalumbo, E., Capozziello, S., Determination of the Intrinsic Luminosity Time Correlation in the X-Ray Afterglows of Gamma-Ray Bursts, 2013, Mon. Not. R. Astron. Soc. , 436, 1;

17. Rowlinson, A., et al., Constraining properties of GRB magnetar central engines using the observed plateau luminosity and duration correlation, 2014, Mon. Not. R. Astron. Soc. , 443, 2, 1779; 
18. Dainotti, M. G., et al., A Study of the Gamma-Ray Burst Fundamental Plane, 2017, Astrophys. $J .$, 848, 88, 2017;

19. Piran, T, The physics of gamma-ray bursts, Rev of Modern Physics, 2004, 76, 4;

20. Xiao, L. \& Schaefer, Estimating Redshifts for Long Gamma-Ray Bursts B.E., 2009, Astrophys. J., 707, 387.

21. Willingale, R., et al., Testing the Standard Fireball Model of Gamma-Ray Bursts Using Late X-Ray Afterglows Measured by Swift, 2007, Astrophys. J., 662, 1093.

22. D'Agostini, G., Fits, and especially linear fits, with errors on both axes, extra variance of the data points and other complications, 2005, arXiv:physics/0511182;

23. Scolnic, D. M., et al., The Complete Light-curve Sample of Spectroscopically Confirmed SNe Ia from Pan-STARRS1 and Cosmological Constraints from the Combined Pantheon Sample, 2018, Astrophys. J., 859, 2, 101;

24. Planck Collaboration, Planck 2018 results. VI. Cosmological parameters, 2018, arXiv:1807.06209;

Acknowledgements This work made use of data supplied by the UK Swift Science Data Centre at the University of Leicester. We are grateful to S. Savastano for helping in writing the python codes. We are grateful to B. De Simone for the help in the analysis of the jet opening angle and of the SNe's likelihood, and to G. Srinivasaragavan, R. Wagner, L. Bowden, Z. Nguyen for the help in the light curves parameters fitting. M.G.D ackowledges the support from the American Astronomical Society Chretienne Fellowship 
and from Miniatura 2. J.F is grateful for the support of the United States Department of Energy in funding the Science Undergraduate Laboratory Internship (SULI) program. We are particularly grateful to Dr. Cuellar for managing the SULI program this summer 2020. This work is supported by JSPS Grants-in-Aid for Scientific Research "KAKENHI” (A: Grant Number JP19H00693). SN also acknowledges the support from Pioneering Program of RIKEN for Evolution of Matter in the Universe (r-EMU). NF acknowledges the support from UNAM-DGAPA-PAPIIT through grant IA102019.

Declaration The authors declare that they have no competing financial interests.

Correspondence Correspondence and requests for materials should be addressed to M.G.D. (email list: mdainott@stanford.edu).

\section{Methods}

\section{The sample Selection}

We analyzed the light curves from 2005 January until 2019 August taken from the Swift web page repository, ${ }^{4}$ and we derived the spectral parameters following ${ }^{4}$.

We fit this sample with the W07 functional form for $f(t)$ which reads as follows:

$$
f(t)= \begin{cases}F_{i} \exp \left(\alpha_{i}\left(1-\frac{t}{T_{i}}\right)\right) \exp \left(-\frac{t_{i}}{t}\right) & \text { for } t<T_{i} \\ F_{i}\left(\frac{t}{T_{i}}\right)^{-\alpha_{i}} \exp \left(-\frac{t_{i}}{t}\right) & \text { for } t \geq T_{i}\end{cases}
$$

\footnotetext{
${ }^{4}$ http://www.swift.ac.uk/burst_analyser
} 
modeled for both the prompt (index ' $\mathrm{i}=p$ ') $\gamma$ - ray and initial X-ray decay and for the afterglow (' $\mathrm{i}=X$ '), so that the complete light curve $f_{\text {tot }}(t)=f_{p}(t)+f_{X}(t)$ contains two sets of four free parameters $\left(T_{i}, F_{i}, \alpha_{i}, t_{i}\right)$, where $\alpha_{i}$ is the temporal PL decay index and $T_{i}$ is the end time of the prompt and the plateau emission, respectively, while the time $t_{i}$ is the initial rise timescale. The transition from the exponential to PL occurs at the point $\left(T_{i}, F_{i} e^{-t_{i} / T_{i}}\right)$, where the two functions have the same value.

After we gather these parameters we proceed with the computation of the peak prompt luminosity at 1 second, $L_{\text {peak }}$, and the luminosity at the end of the plateau emission, $L_{X}$, using the following equation:

$$
L=4 \pi D_{L}^{2}(z) F\left(E_{\min }, E_{\max }, T_{X}^{*}\right) \cdot K
$$

where $F=F_{X}, F_{\text {peak }}$, with $F_{X}$ the measured X-ray energy flux at the end of the plateau phase and $F_{\text {peak }}$ the measured $\gamma$-ray energy flux over a $1 \mathrm{~s}$ interval $\left(\mathrm{erg}_{\mathrm{cm}}^{-2} \mathrm{~s}^{-1}\right)$ in the peak of the prompt emission, $D_{L}(z)$ is the distance luminosity for a given redshift $z$, assuming a flat $\Lambda \mathrm{CDM}$ cosmological model with energy equation of state $w=-1, \Omega_{M}=0.3$ and $H_{0}=70 \mathrm{Km} \mathrm{s}^{-1}$ $M p c^{-1}, T_{X}^{*}$ is the rest frame time at the end of the plateau and $K$ is the $K$-correction for the cosmic expansion defined in Eq. 5 shown below ${ }^{25}$. For Swift GRBs, $K$ is calculated in the following way 25

$$
K=\frac{\int_{E_{\min } /(1+z)}^{E_{\max } /(1+z)} \Phi(E) d E}{\int_{E_{\min }}^{E_{\max }} \Phi(E) d E}
$$

where $\Phi(E)$ is the functional form for the spectrum, for which we assume either a PL for the plateau phase and a cutoff power law (CPL) for the prompt emission. In a few cases when the CPL for the prompt emission is not a viable fit we use the PL. 
To further create a sample with more homogeneous spectral features, and hence restricting the analysis to a more uniform class of objects, we consider the GRBs for which the spectrum computed at $1 \mathrm{~s}$ has a smaller $\chi^{2}$ for the CPL fit than for a PL. Specifically, following ${ }^{26}$, when the $\chi_{C P L}^{2}-\chi_{P L}^{2}<6$, either a PL or a CPL can be chosen, since the goodness of the fit is equivalent, and in these cases we here chose the CPL. In addition, for all GRBs for which the CPL was absent or the $\chi^{2}$ satisfies this criterion, there is no substantial difference in the spectral fitting results if one considers either a PL or a CPL. We have discarded six GRBs that were better fitted with a black body model than with a PL or a CPL.

From a total sample of 214 GRBs presenting plateaus, we choose a subsample by considering strict data quality and the following morphology requirements:

- the beginning of the plateau must have at least five data points;

- the plateau must not be too steep (the angle of the plateau has to be less than $41^{\circ}$ );

- $T_{X}$ must not be inside a large gap of the observed data;

- The plateau duration must be (>500 s)and the plateau must not contain flares or bumps.

The light curves with these features create a sample of 50 platinum GRBs. It is important to note that the criteria defining our sample are objectively determined before the construction of the correlations sought; sample cuts are introduced strictly following either data quality or physical class constraints. The furthest GRB belonging to the platinum sample is found at $z=5$. 


\section{The Angle Computation}

The problem is that there are only a few observed jet opening angles, $\theta_{j e t}$. To overcame this issue we use an indirect method to estimate these quantities built upon the method of Pescalli ${ }^{2} 7$, which, in turn, is based on the ratio between the $E_{\text {peak }}-E_{\text {iso }}{ }^{2} 8$ and the $E_{\text {peak }}-E_{\gamma, \text { iso }}$ correlations ${ }^{2} 9$, where $E_{\text {peak }}, E_{\text {iso }}$ and $E_{\gamma, \text { iso }}=E_{\text {iso }} \times(1-\cos \theta)$ are the peak of the $\nu F_{\nu}$ spectrum, the isotropic energy and the collimated energy, respectively. All these quantities are computed for the prompt emission. The Pescalli method involves prompt emission variables, and we improve upon this using the fundamental plane relation, which also considers plateau emission variables.

More specifically, we assume, analogously to the Pescalli derivation, that the fundamental plane relation is a general correlation, but the scatter that we infer for it is not only due to the intrinsic physical scatter, at least not for the majority of it, but also due to the fact we are not correcting the luminosities for the jet opening angle.

Thus, we add a correction to the Pescalli estimation of the angle due to the luminosity at the end of the plateau emission. This correction, which we call vertical shift, $s$, represents how much a jetted emission would influence the determination of the true luminosity.

We calculate, as a first approximation, the values of the jet opening angle using the method described in ${ }^{27}$ with the following equation:

$$
\log \left(1-\cos \left(\theta_{\text {jet }}\right)=C+\xi \times \log L_{\text {iso }}\right.
$$

where $\mathrm{C}$ is a normalization constant, which here is defined as the minimum value of $L_{\text {iso }}=$ 
$E_{i s o} / T_{90}$ for a given sample, and $\xi=0.3$, following ${ }^{27}$. We compute $E_{\text {iso }}$ as the following:

$$
E_{i s o}=4 \pi D_{L}^{2}(z) S\left(E_{\min }, E_{\max }, T_{X}^{*}\right) \cdot K
$$

where $S$ is the fluence taken from the Third Swift BAT Gamma-Ray Burst Catalog ${ }^{3} 0$ and $K$ is the $K$-correction already defined above.

To give generality to our method, we have simulated all the parameters involved in our fitting and in Eq. 6. These parameters are derived by the best fit distributions computed from the observed data of the platinum sample.

We use a new approach to compute the jet opening angle refining Eq. 6 by using the vertical shift from the three-dimensional fundamental plane relation. We can leverage from the previous Eqs., for which $1-\cos \left(\theta_{j e t}\right) \propto L_{i s o}$, and from the fact that, observationally, $\log L_{i s o} \propto \beta \times$ $\log L_{\text {peak }}$. Since the fundamental plane relates $\log L_{\text {peak }}$ with $\log L_{X}$ and $\log T_{X}^{*}$, we can add to the derivation of Pescalli ${ }^{27}$ the information given by the fundamental plane correlation in the following way:

$$
\log \left(1-\cos \left(\theta_{j e t}\right)\right)=C^{\prime}-\xi^{\prime} \times \log L_{\text {peak }}+\alpha \times s
$$

where $\alpha \times s$ is the correction factor that we apply to the Pescalli formula, see Eq. 6, and $C^{\prime}$ and $\xi^{\prime}$ are the new coefficients obtained by fitting the Pescalli angle with the vertical shift and $L_{\text {peak }}$. The vertical shift is the defined as

$$
s=\log L_{X}-\left(a_{\text {sim,platinum }} \times \log T_{X, \text { sim }}^{*}+b_{\text {sim,platinum }} \times \log L_{\text {peak,sim }}+C_{0, \text { sim,platinum }}\right)
$$


where $a_{\text {sim,platinum }}, b_{\text {sim,platinum }}$ and $C_{0, \text { sim,platinum }}$ are the best fit parameters of the simulated isotropic fundamental plane relation for the platinum sample (Eq. 1), $L_{\text {peak,sim }}$ and $T_{X, \text { sim }}^{*}$ are the simulated peak prompt luminosity and end time of the plateau emission of the GRBs belonging to the platinum sample. We stress again that we use simulations to give generality to the definition of the vertical shift. More specifically, to guarantee the generality of this equation, we have simulated the best fit distribution parameters of the observed data of the platinum sample 10000 times. Then, Eq. 9 is substituted into Eq. 8 to compute the coefficients $C^{\prime}, \xi^{\prime}$ and $\alpha$. We obtain these coefficients by fitting Eq. 8 and repeat the fitting 10000 times to compute the mean of these best fit parameters as the final values. This procedure will guarantee that the values computed are statistically meaningful over repeated iterations to correctly compute the errors associated with them. To compute the jet opening angle for each GRB in our sample, we use Eq. 8 to have a one to one correspondence of each GRB with its jet opening angle.

We then substitute $(1-\cos \theta)$ in the jetted fundamental plane, see Eq. 2 and, thus, we obtain a new set of best fit parameters with a much smaller intrinsic scatter with respect to the isotropic fitting.

Indeed, from Figs. 3 and 4 , we see that the fundamental plane for the platinum sample corrected for the jet opening angle (its projection in 2D is shown in the lower panel of Fig. 3) leads to a remarkable reduction of $\sigma_{i n t}$ compared to the isotropic fundamental plane (its $2 \mathrm{D}$ projection is shown in the upper panel of Fig. 3). In fact, we reduce $\sigma_{i n t}$ of $95 \%$ from $0.34 \pm 0.04$ to $0.017 \pm 0.010$. The contour plots of the best fit parameters are shown in Fig. 4. 
To find these best fitting parameters we use the D'Agostini ${ }^{22}$ Bayesian method, which takes into account the error bars on all the axes and it also includes $\sigma_{i n t}$. Uncertainties are always quoted in $1 \sigma$.

\section{The 3D Relation for platinum GRBs with correction for $\theta_{j e t}$ as a cosmological tool.}

Here we summarize the method used to obtain cosmological parameters by using the 3D fundamental plane with the platinum sample by introducing the $\theta_{j e t}$ correction together with SNe Ia data. The values of $\theta_{\text {jet }}$ are derived from Eq. 8.

For the flat $\Lambda \mathrm{CDM}$ cosmological model, in which we have neglected the radiation contribution, with energy equation of state $w=-1$ and $H_{0}=70 \mathrm{Km} \mathrm{s}^{-1} \mathrm{Mpc}^{-1}$, the luminosity distance used in Eqs. 4 and 7 is written as follows:

$$
D_{L}(z)=(1+z) \frac{c}{H_{0}} \int_{0}^{z} \frac{d z^{\prime}}{\sqrt{\Omega_{M}\left(1+z^{\prime}\right)^{3}+\left(1-\Omega_{M}\right)}}
$$

where $c$ is the speed of light and $z$ is the redshift of the GRB for which we are computing the distance. We recall that in our Bayesian approach, we let $\Omega_{M}$ vary simultaneously with the other variables, so that we do not incur into the so-called circularity problem. This means that we derive each time the best fit parameters for the three-dimensional relation corrected for the jet opening angle for each given value of $\Omega_{M}$. The same is true when we fix $\Omega_{M}=0.3$ and let vary $H_{0}$. Instead, when we fix $\Omega_{M}=0.3, H_{0}=70 \mathrm{Km} \mathrm{s}^{-1} M p c^{-1}$ and we consider a $w \mathrm{CDM}$ model for which we let vary the parameter $w$ (without considering a dependency on the redshift), the 
luminosity distance becomes: ${ }^{31}$

$$
D_{L}(z)=(1+z) \frac{c}{H_{0}} \int_{0}^{z} \frac{d z^{\prime}}{\sqrt{\Omega_{M}\left(1+z^{\prime}\right)^{3}+\left(1-\Omega_{M}\right)\left(1+z^{\prime}\right)^{3(1+w)}}} .
$$

In our cosmological computations, we use both the GRBs belonging to the platinum sample and the SNe Ia data of the Pantheon sample ${ }^{23}$. To evaluate the best cosmological parameters underlying our universe, we make use of the distance moduli, $\mu_{o b s, S N e}$, derived from the observations of SNe Ia taken directly from ${ }^{23}$. Regarding the $\mu_{o b s, G R B s}$ of GRBs, we derive it performing algebraic manipulations on the fundamental plane relation corrected for the jet opening angle that lead to the following formula:

$$
\mu_{o b s, G R B s}=5\left(b_{1} \log F_{p, c o r}+a_{1} \log F_{a, c o r}+c_{1}+d_{1} \log T_{X}^{*}\right)+25
$$

where $\log F_{p, c o r}$ and $\log F_{a, c o r}$ are the fluxes in the prompt and afterglow emission corrected for the $K$-correction and the jet opening angle. We then compare both $\mu_{o b s, G R B s, S N e}$ with the theoretical $\mu_{t h}$, defined as follows (with the appropriate dimensional units):

$$
\mu_{t h}=5 \cdot \log _{10} D_{L}\left(z, \Omega_{M}, H_{0}, w\right)+25
$$

We have combined the two likelihoods for our Bayesian approach, one related to the GRBs while the other to the SNe Ia. The total likelihood is:

$$
\mathcal{L}(G R B s)+\mathcal{L}(S N e)=\sum_{i}\left(\log \left(\frac{1}{\sqrt{2 \pi} \sigma_{\mu, i}}\right)-\frac{1}{2}\left(\frac{\mu_{t h, G R B, i}-\mu_{o b s, G R B, i}}{\sigma_{\mu, i}}\right)^{2}\right)-\frac{1}{2}\left(\left(\mu_{t h, S N e}-\mu_{o b s, S N e}\right)^{T} \times\right.
$$


where the first term is the likelihood related to GRBs' distance moduli ${ }^{16,32}$, and the second refers to the likelihood of SNe Ia data, where $C_{i n v}$ is the inverse of the covariance matrix of the $\mathrm{SNe}$ data 23.

We have computed the cosmological parameters using only SNe Ia data as well, to see if adding GRBs would confirm the results and to what extent we could improve the precision on the cosmological parameters. To check the consistency and the reliability of our results, we use the SNe Ia Pantheon sample alone in four cases. In the $\Lambda$ CDM model, for case (1) varying $\Omega_{M}$ and fixing $H_{0}$ we obtain $\Omega_{M}=0.298 \pm 0.010$, see Fig. 5; for case (2) varying $H_{0}$ and fixing $\Omega_{M}$ we obtain $H_{0}=69.96 \pm 0.15 \mathrm{Km} \mathrm{s}^{-1} \mathrm{Mpc}^{-1}$, see Fig. 6; for case (3) varying both $H_{0}$ and $\Omega_{M}$ we obtain $\Omega_{M}=0.300 \pm 0.020$ and $H_{0}=69.97 \pm 0.33 \mathrm{Km} \mathrm{s}^{-1} \mathrm{Mpc}^{-1}$, see Fig. 7; for case (4) in the $w$ CDM model varying $\mathrm{w}$ and fixing $\Omega_{M}$ and $H_{0}$ we obtain $w=-1.066 \pm 0.019$, see Fig. 8 . We then have tested the same four cases mentioned above for the $\mathrm{SNe}+\mathrm{GRB}$ data: in case (1) we derive $\Omega_{M}=0.299 \pm 0.009$, see Fig. 9; in case (2) we obtain $H_{0}=69.97 \pm 0.13 \mathrm{Km} \mathrm{s}^{-1} \mathrm{Mpc}^{-1}$, see Fig. 2; in case (3) we derive $\Omega_{M}=0.305 \pm 0.022$ and $H_{0}=69.89 \pm 0.34 \mathrm{Km} \mathrm{s}^{-1} \mathrm{Mpc}^{-1}$, see Fig. 10; in case (4) we obtain $w=-1.006 \pm 0.019$, see Fig. 11. All the results for SNe Ia alone and GRBs + SNe Ia together are summarized in Table 1, where we have explicitly indicated the cases' number in each row. We can clearly see that the introduction of GRBs does reduce the errors on the cosmological parameters in cases (1) and (2). We have compared our results with the ones found in the literature using other cosmological probes: for instance, ${ }^{23}$ obtained $\Omega_{M}=0.284 \pm 0.012$ for 
the $\Lambda$ CDM model, while for the $w \mathrm{CDM}$ one, they obtained $w=-1.026 \pm 0.041$. These results are consistent with the ones derived by our computations in $1 \sigma$. We here stress that by adding GRBs to $\mathrm{SNe}$ Ia for case (2), see Table 1 , in which we vary $H_{0}$, we reached a $15.4 \%$ decrease in the error on $H_{0}$, while for case (1) we obtain a $11 \%$ decrease in the scatter for $\Omega_{M}$.

The crucial point of our results is that we are able to obtain compatible cosmological parameters with better precision in some cases by also using GRBs and with the great advantage of using cosmological probes which are observed at very high redshift, in this case of our sample up to redshift 5 .

\section{Additional References}

25. Bloom, The Prompt Energy Release of Gamma-Ray Bursts using a Cosmological k-Correction, 2001, Astrophys. J., 121, 6, 2879;

26. Sakamoto, T., et al., The Second Swift Burst Alert Telescope Gamma-Ray Burst Catalog, 2011, Astrophys. J. Supp., 195, 2.

27. Pescalli, A., et al., Luminosity function and jet structure of Gamma-Ray Burst, 2015, Mon. Not. R. Astron. Soc. , 447, 1911

28. Amati, L., et al., Intrinsic spectra and energetics of BeppoSAX Gamma-Ray Bursts with known redshifts, 2002, Astron. Astrophys, 390, p.81-89; 
29. Ghirlanda, G., Ghisellini, G., Lazzati, D., The Collimation-corrected Gamma-Ray Burst Energies Correlate with the Peak Energy of Their $\nu F_{\nu}$ Spectrum, 2004, Astrophys. J., 616, 1;

30. Lien, A., et al., The Third Swift Burst Alert Telescope Gamma-Ray Burst Catalog, 2016, Astrophys. J., 829, 1, 7;

31. Tripathi, A., Sangwan, A., Jassal, H. K., Dark energy equation of state parameter and its evolution at low redshift, 2017, J. Cosmol. Astropart. P., 6, id. 12

32. Amati, L., et al., 2019, Addressing the circularity problem in the $E_{p i}-E_{i s o}$ correlation of gamma-ray bursts, 2019, Mon. Not. R. Astron. Soc. Lett., 486, p.L46-L51;

competing financial interests. 


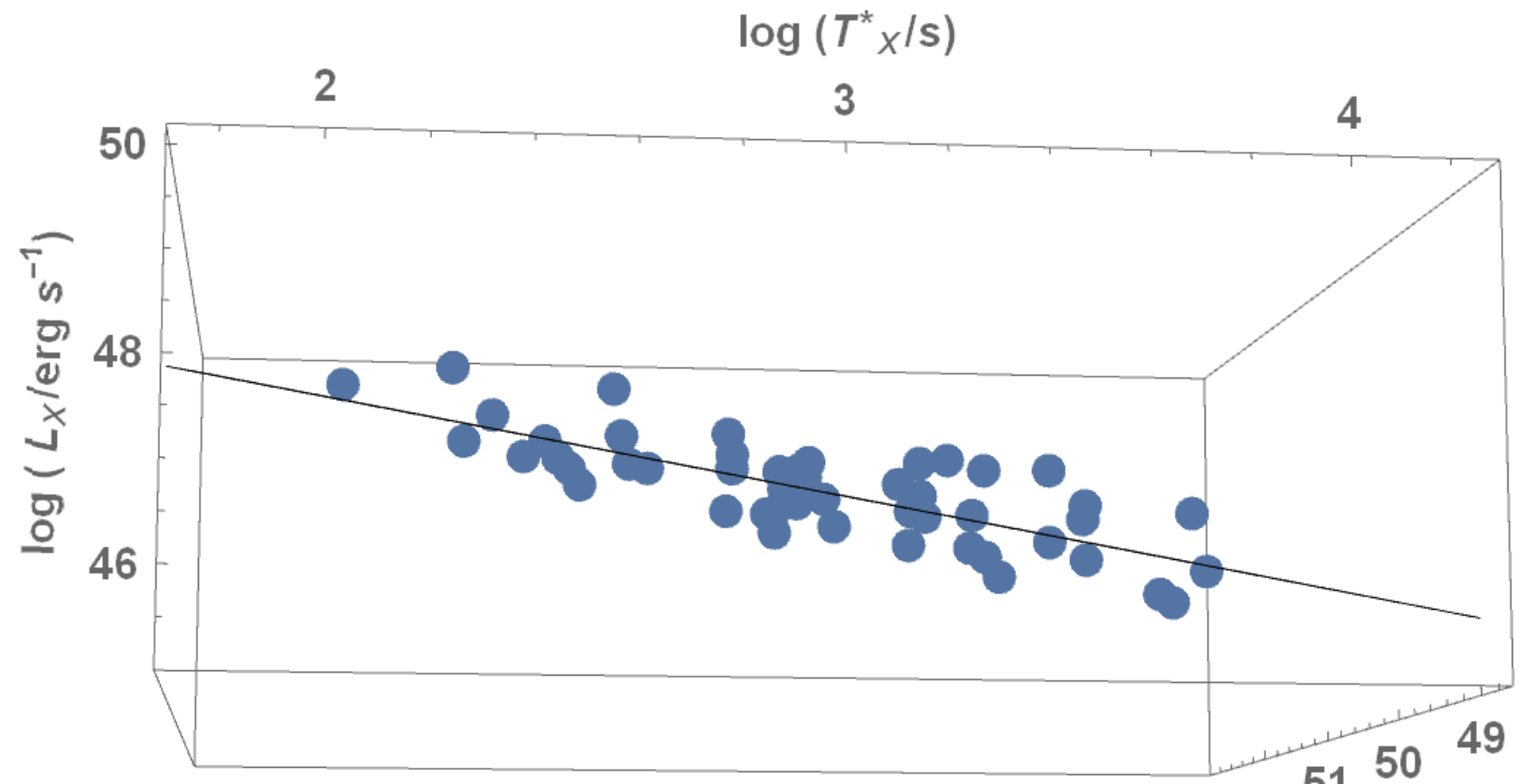

$\log \left(T^{*} \times / s\right)$

$2 \quad 3 \quad 4$

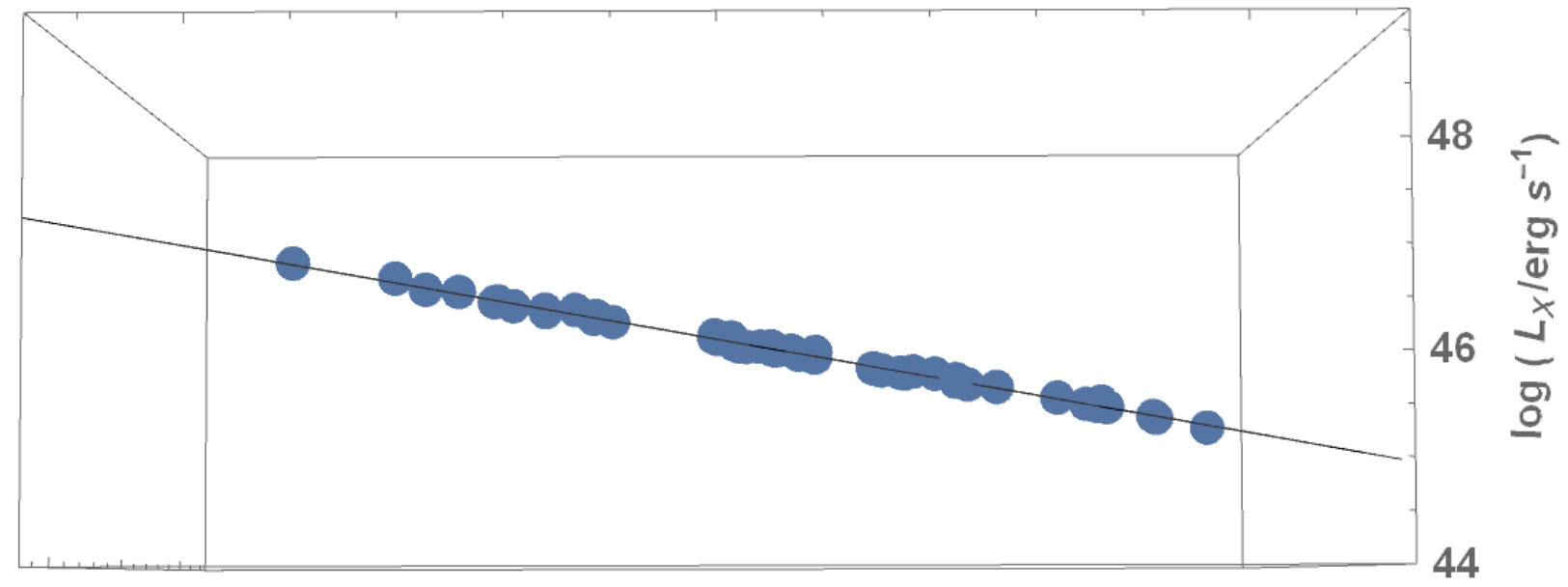

495051

$\log \left(L_{\text {peak }} / \mathrm{erg} \mathrm{s}^{-1}\right)$

Figure 1: Upper panel: the isotropic fundamental plane relation in 3D space between the luminosity at the end of the plateau emission, $\mathrm{L}_{\mathrm{X}}$, the rest frame end time of the plateau emission itself, $\mathrm{T}^{*} \mathrm{x}$, and the peak prompt luminosity, $\mathrm{L}_{\text {peak. }}$ Lower panel: the same fundamental plane relation, but corrected for the jet opening angle in 3D. 


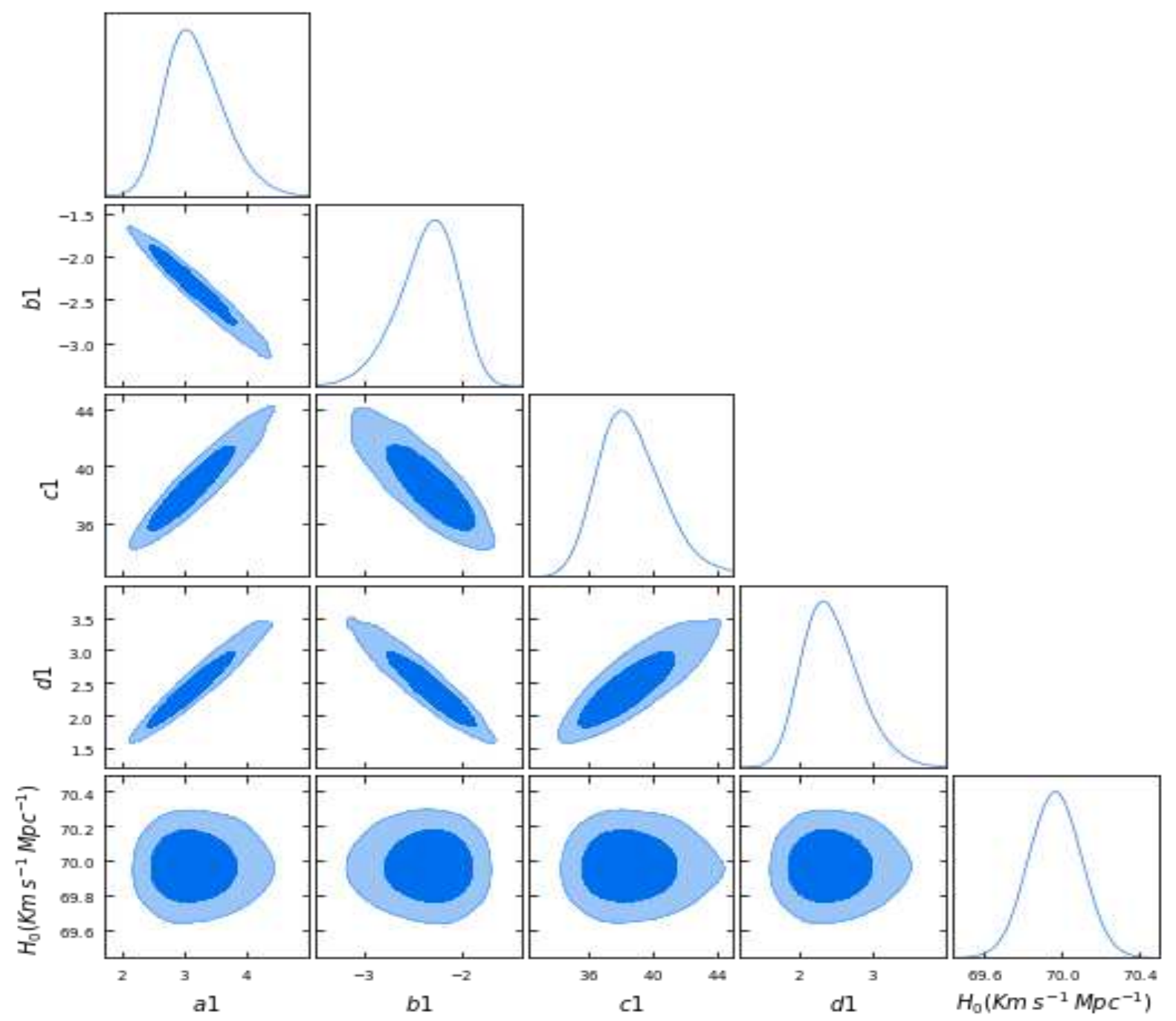

Figure 2: Result of the cosmological fit for $\mathrm{H}_{0}$ considering both GRBs and SNe Ia data and by fixing $\Omega_{\mathrm{M}}=0.3$ at a fiducial value and $\mathrm{w}=-1$. 


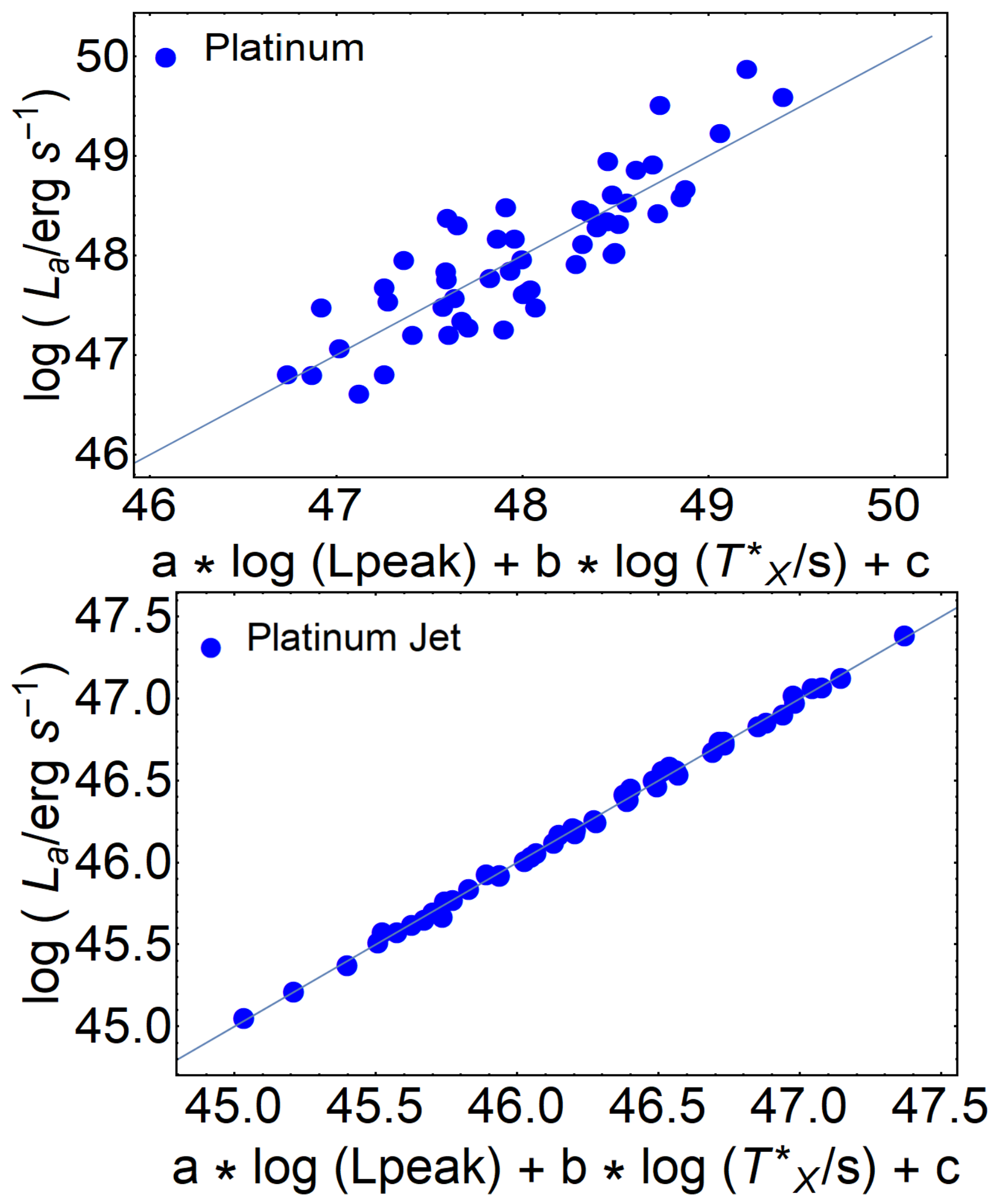

Figure 3: Upper panel: The isotropic fundamental plane relation in the isotropic case projected in a 2D plot. Lower panel: the jetted fundamental plane corrected for the jet opening angle again projected in $2 \mathrm{D}$. 


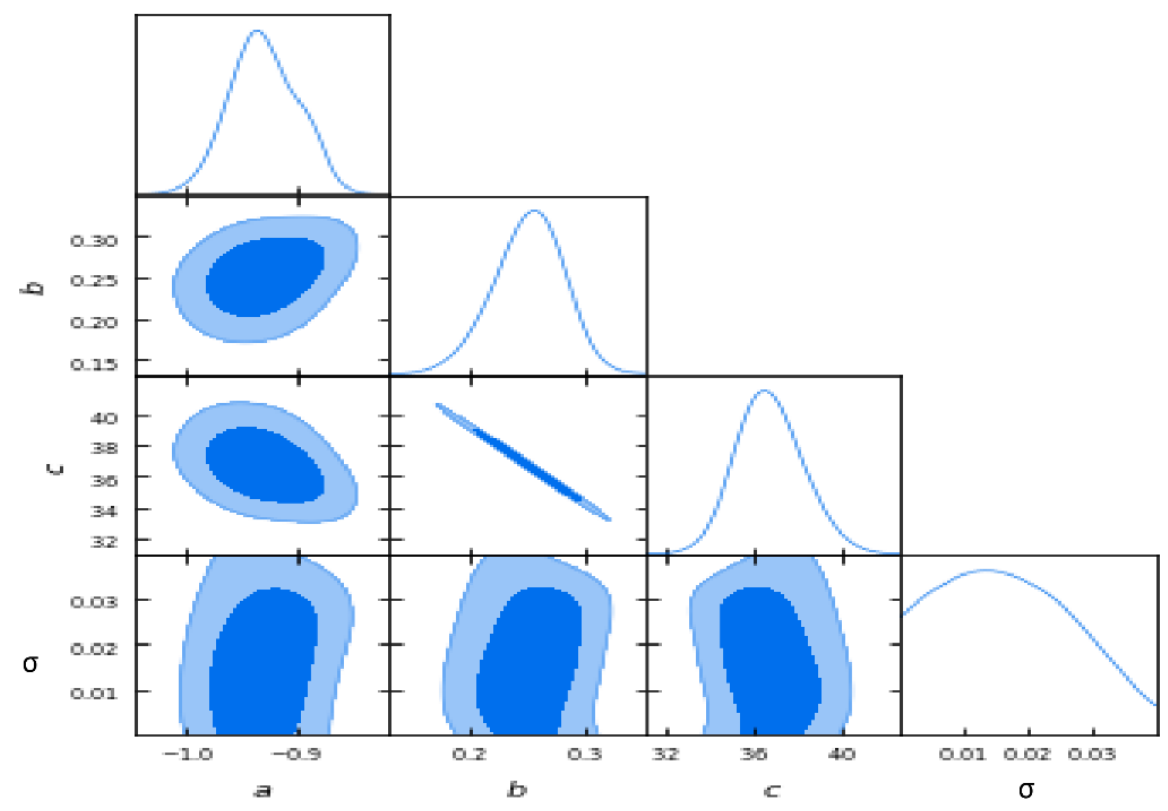

Figure 4: Contour plots at $68 \%$ and $95 \%$ level (the darker blue and the lighter blue, respectively) for the best fit parameters of the jetted fundamental plane of the platinum sample. 


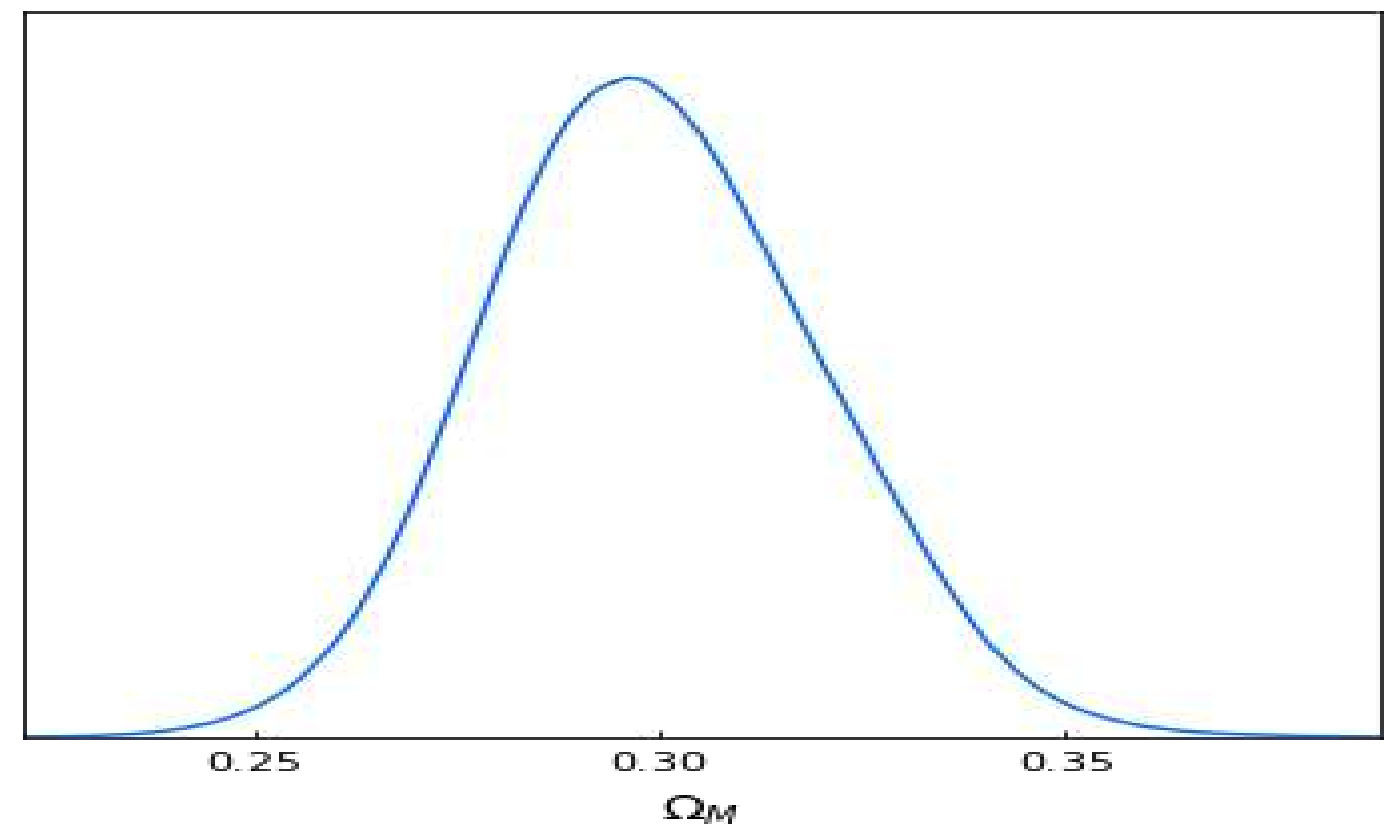

Figure 5: Result of the cosmological fit for $\Omega_{\mathrm{M}}$ considering only SNe Ia data and fixing $\mathrm{H}_{0}=70 \mathrm{Km}$ $\mathrm{s}^{-1} \mathrm{Mpc}^{-1}$ and $\mathrm{w}=-1$ at fiducial values.

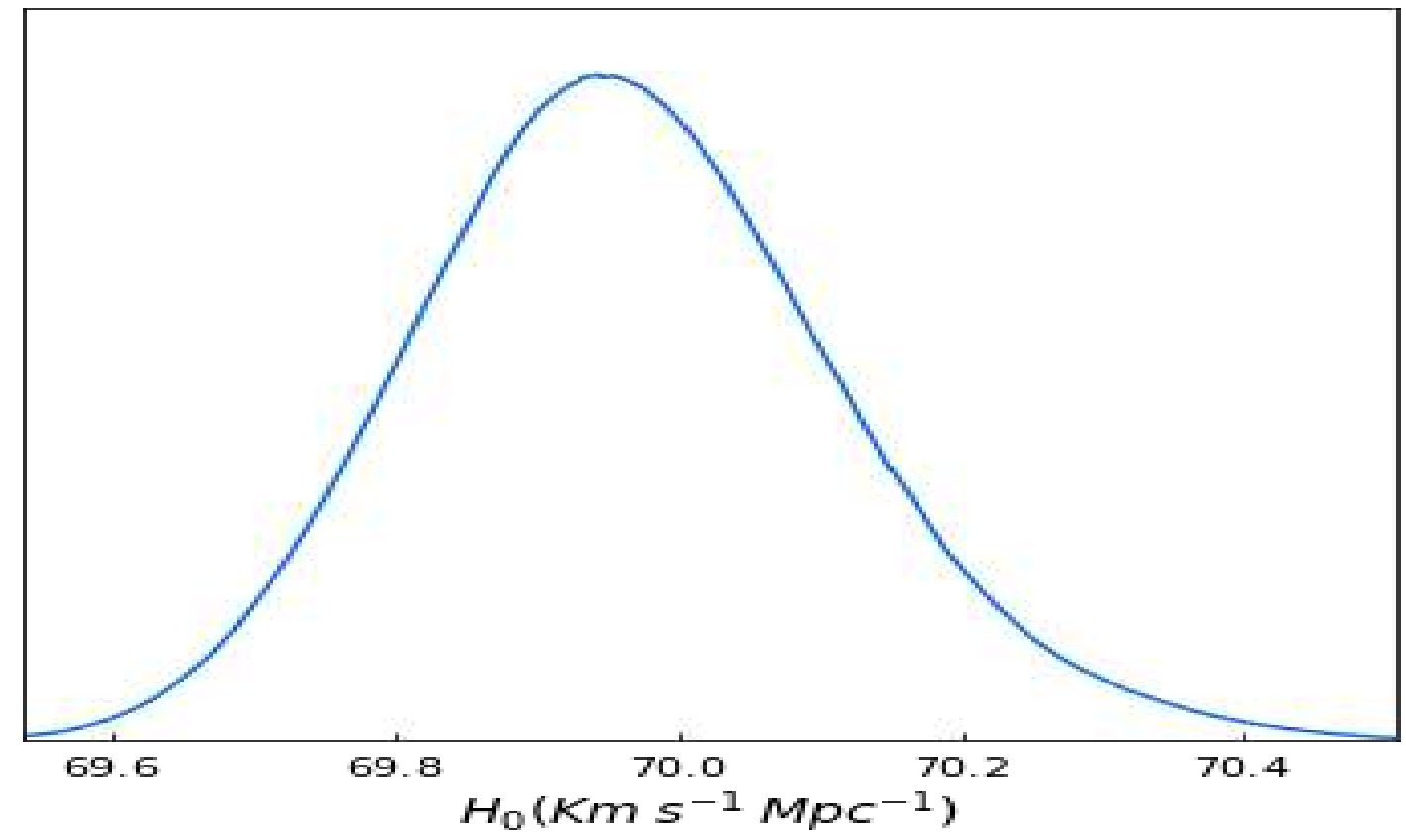

Figure 6: Result of the cosmological fit for $\mathrm{H}_{0}$ considering only SNe Ia data by fixing $\Omega_{\mathrm{M}}=0.3$ and $\mathrm{w}=-1$ at fiducial values. 


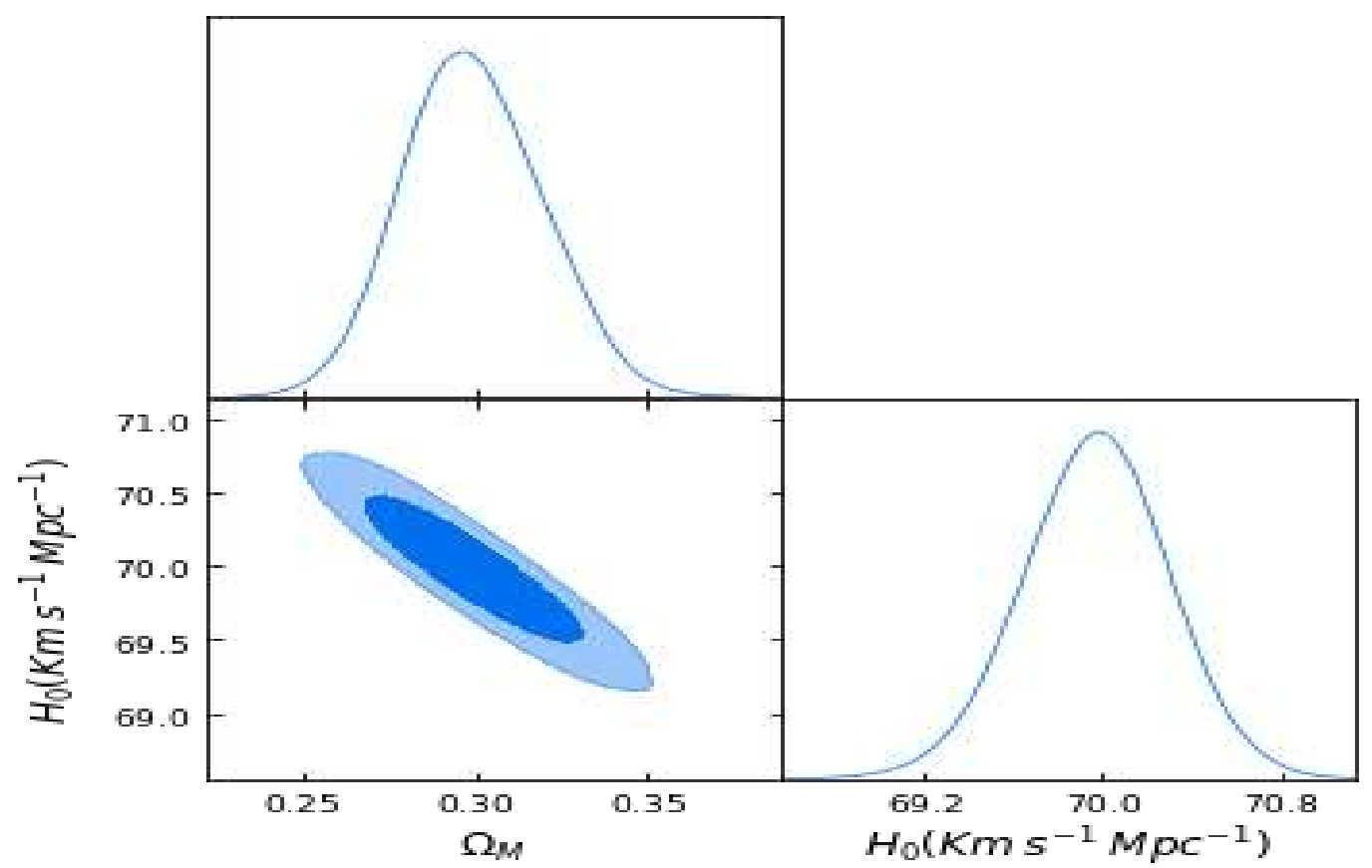

Figure 7: Contour plots of the parameters of only SNe Ia data at 68\% (dark blue contours) and 95\% confidence level (light blue contours) showing the result of the cosmological fit for $\Omega_{\mathrm{M}}$ and $\mathrm{H}_{0}$ fixing $w=-1$.

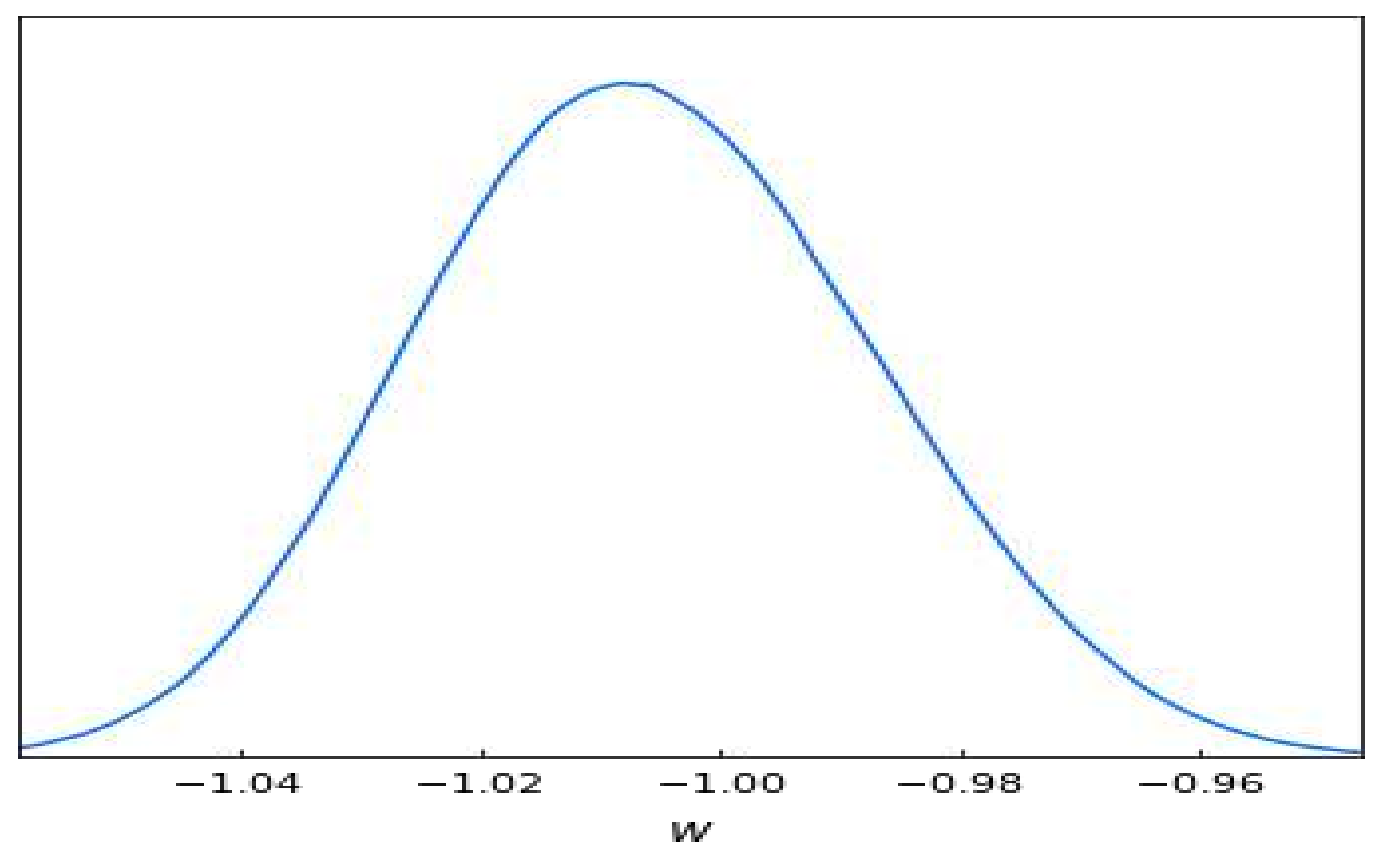

Figure 8: Result of the cosmological fit for w using only SNe Ia data and by fixing $\Omega_{\mathrm{M}}=0.3$ and $\mathrm{H}_{0}=70 \mathrm{Km} \mathrm{s}^{-1} \mathrm{Mpc}^{-1}$ at fiducial values. 


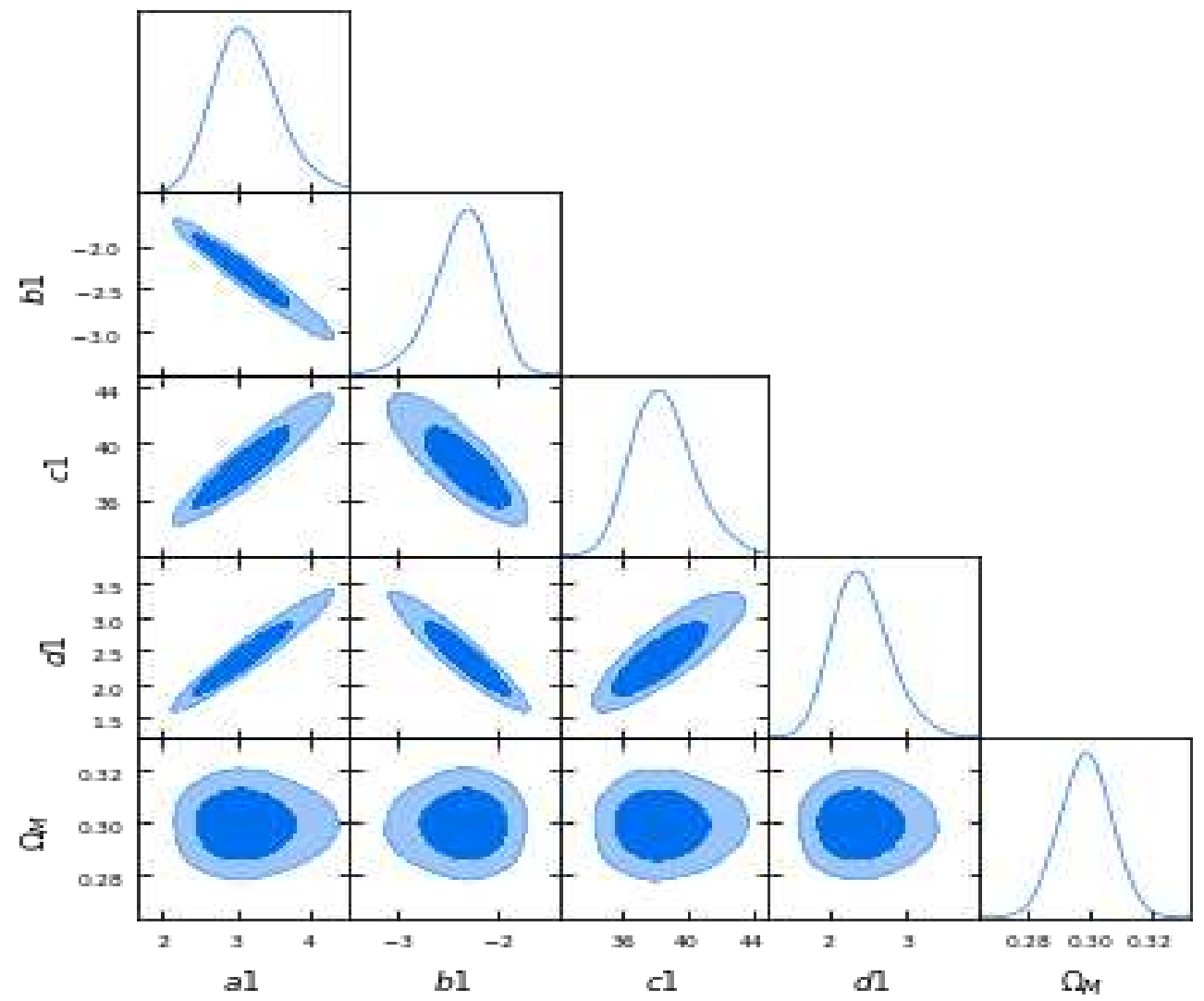

Figure 9: Result of the cosmological fit for $\Omega_{\mathrm{M}}$ considering both GRBs and SNe Ia data and by fixing $\mathrm{H}_{0}=70 \mathrm{Km} \mathrm{s}^{-1} \mathrm{Mpc}^{-1}$ at fiducial value and $\mathrm{w}=-1$. 

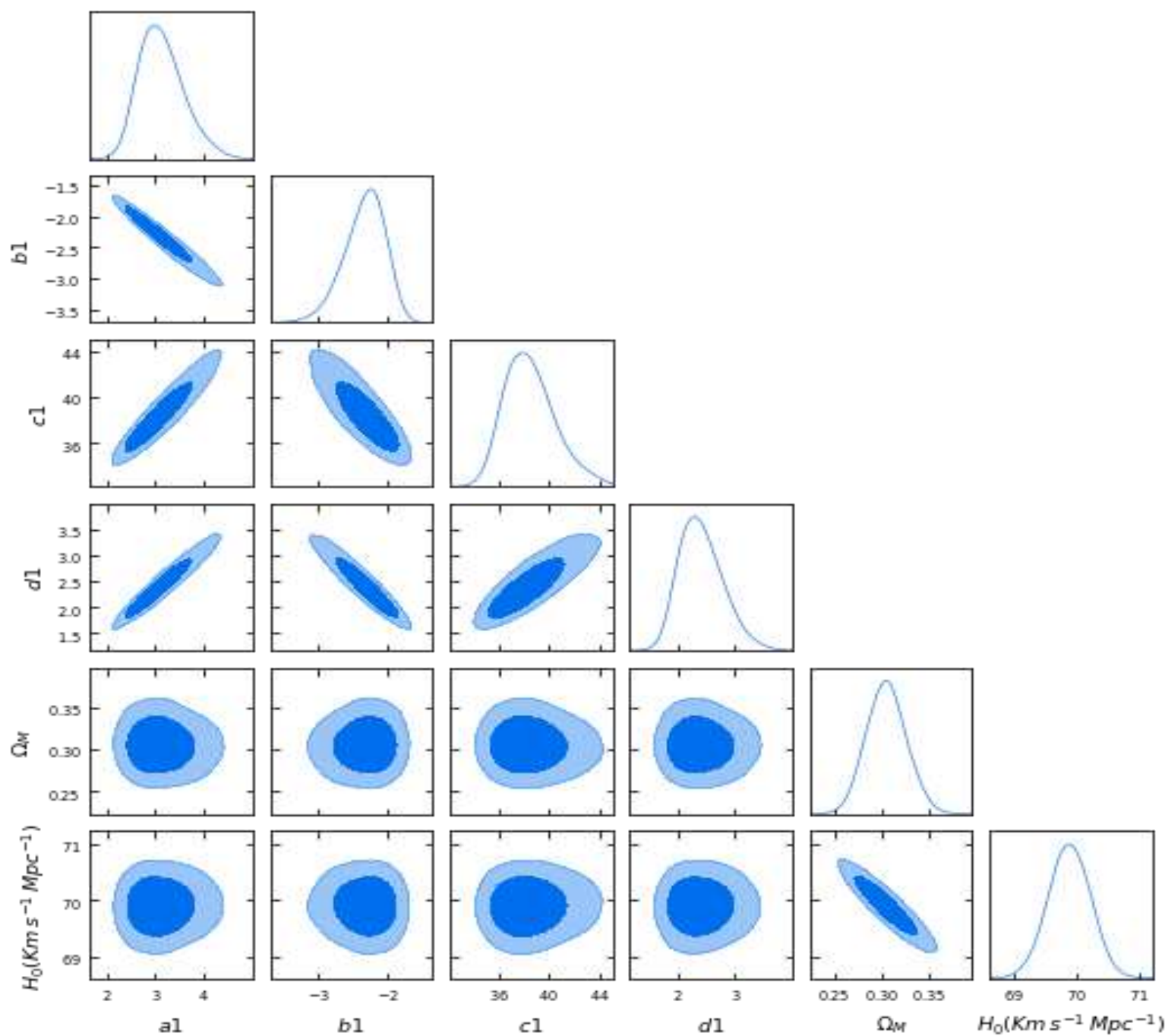

Figure 10: Contour plots of the parameters of the GRB platinum sample and SNe Ia together at $68 \%$ (dark blue contours) and 95\% confidence level (light blue contours) and result of the cosmological fit for $\Omega_{M}$ and $\mathrm{H}_{0}$ considering both GRBs and SNe Ia data. 


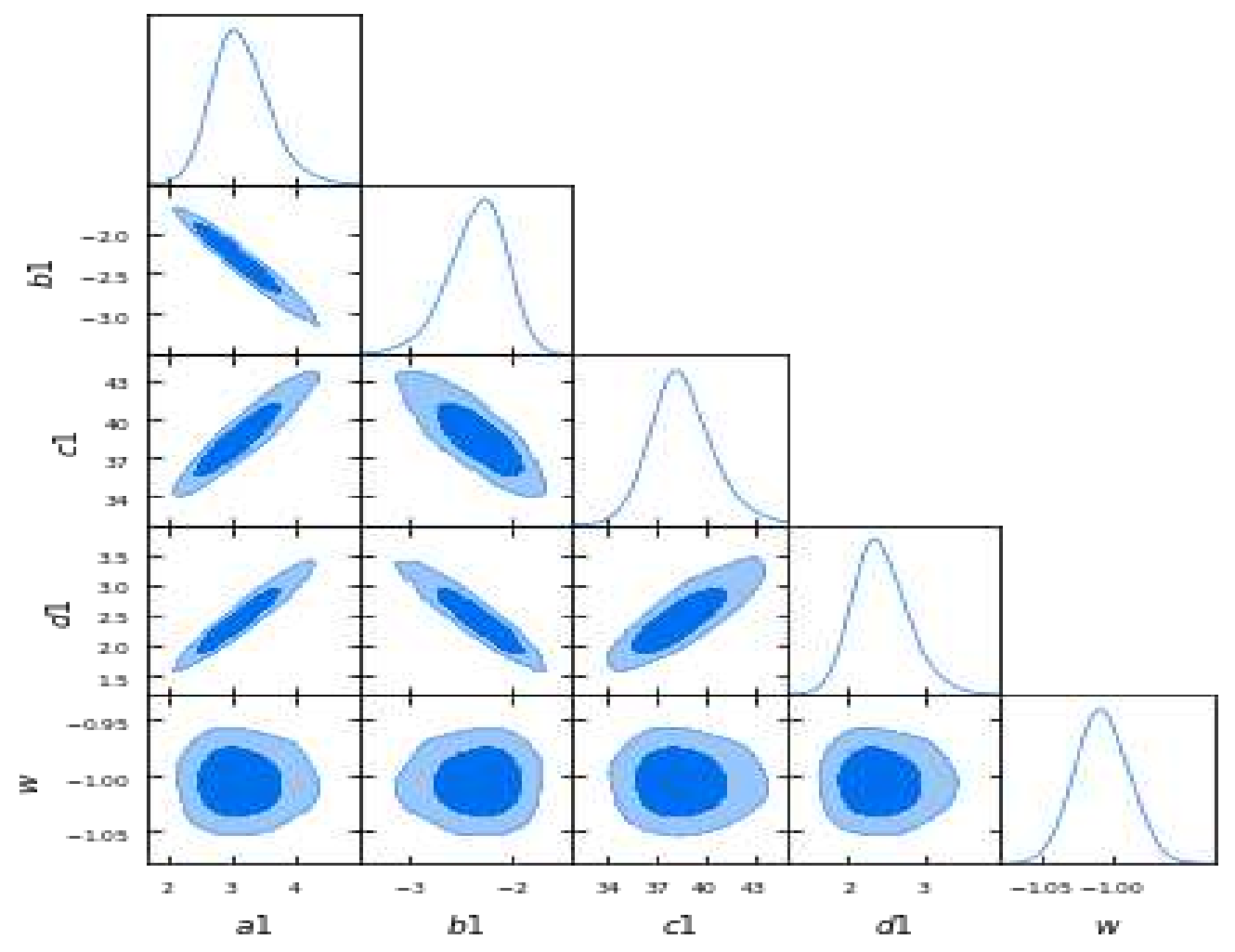

Figure 11: Contour plots of the parameters of the SNe Ia and the GRB platinum sample at $68 \%$ (dark blue contours) and 95\% confidence level (light blue contours) and result of the cosmological fit for w fixing $\Omega_{\mathrm{M}}=0.3$ and $\mathrm{H}_{0}=70 \mathrm{Km} \mathrm{s}^{-1} \mathrm{Mpc}^{-1}$ at fiducial values. 
Figures

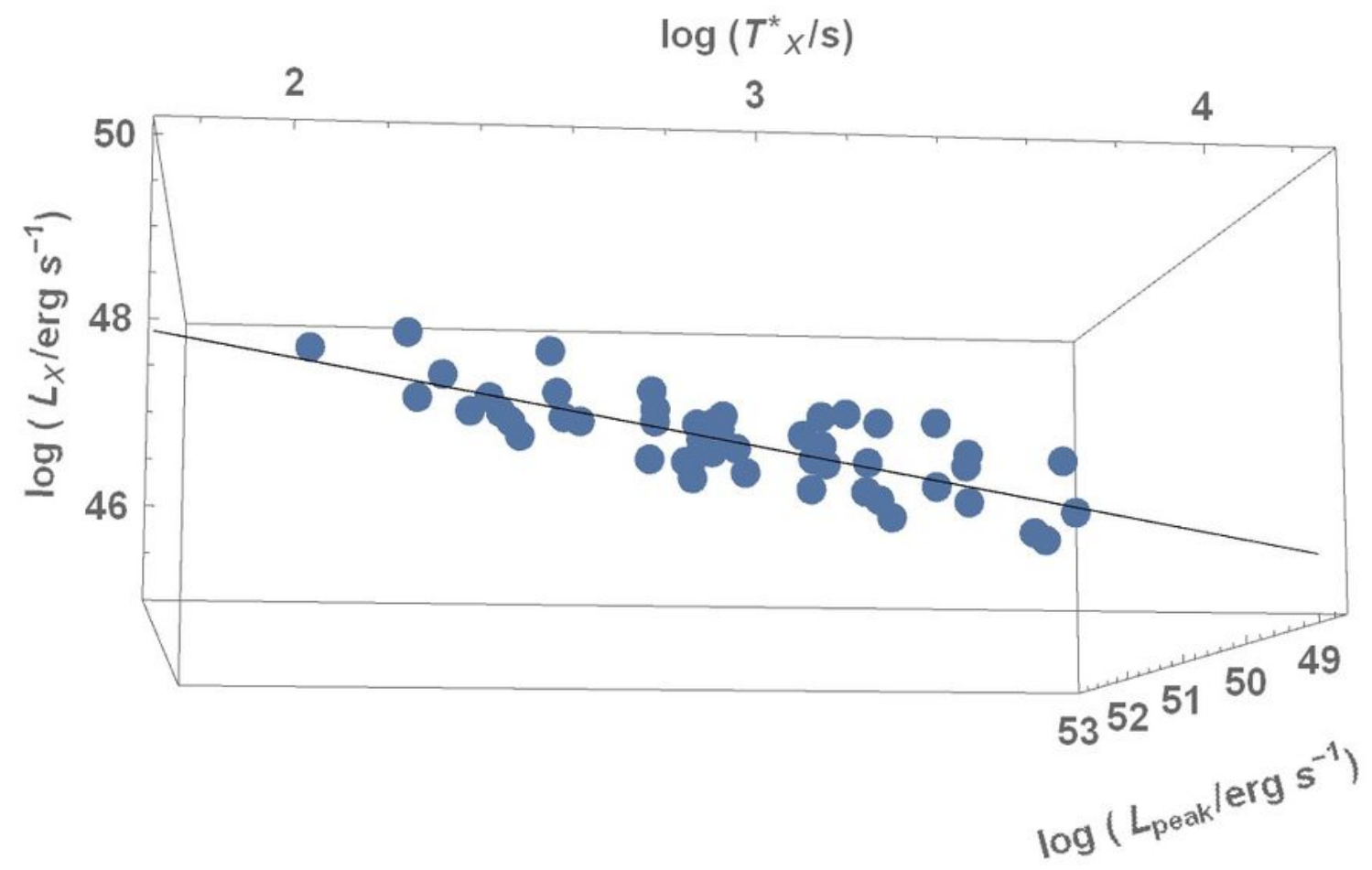

$\log \left(T^{*} \times / \mathbf{s}\right)$

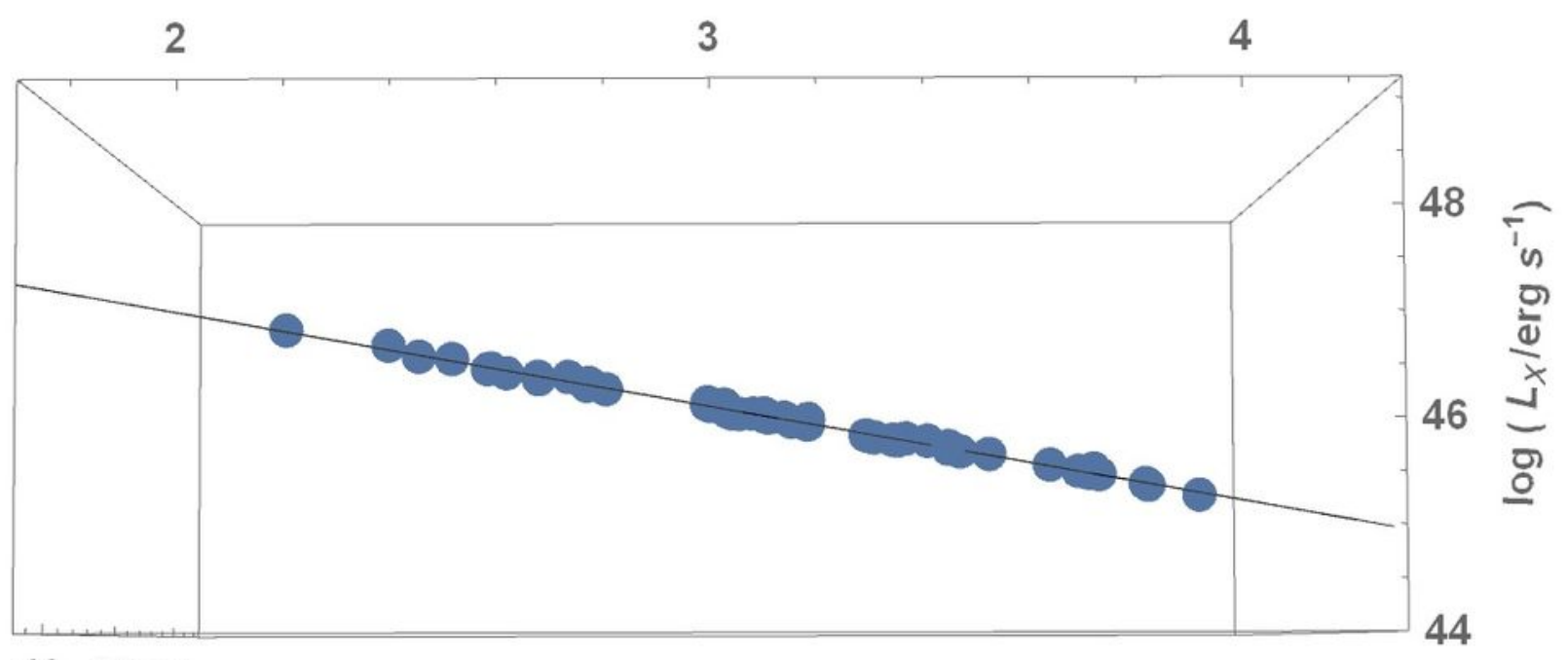

495051

$\log \left(L_{\text {peak }} /\right.$ erg s $\left.{ }^{-1}\right)$

\section{Figure 1}

Upper panel: the isotropic fundamental plane relation in 3D space between the luminosity at the end of the plateau emission, $L X$, the rest frame end time of the plateau emission itself, $T * X$, and the peak 
prompt luminosity, Lpeak. Lower panel: the same fundamental plane relation, but corrected for the jet opening angle in $3 \mathrm{D}$.

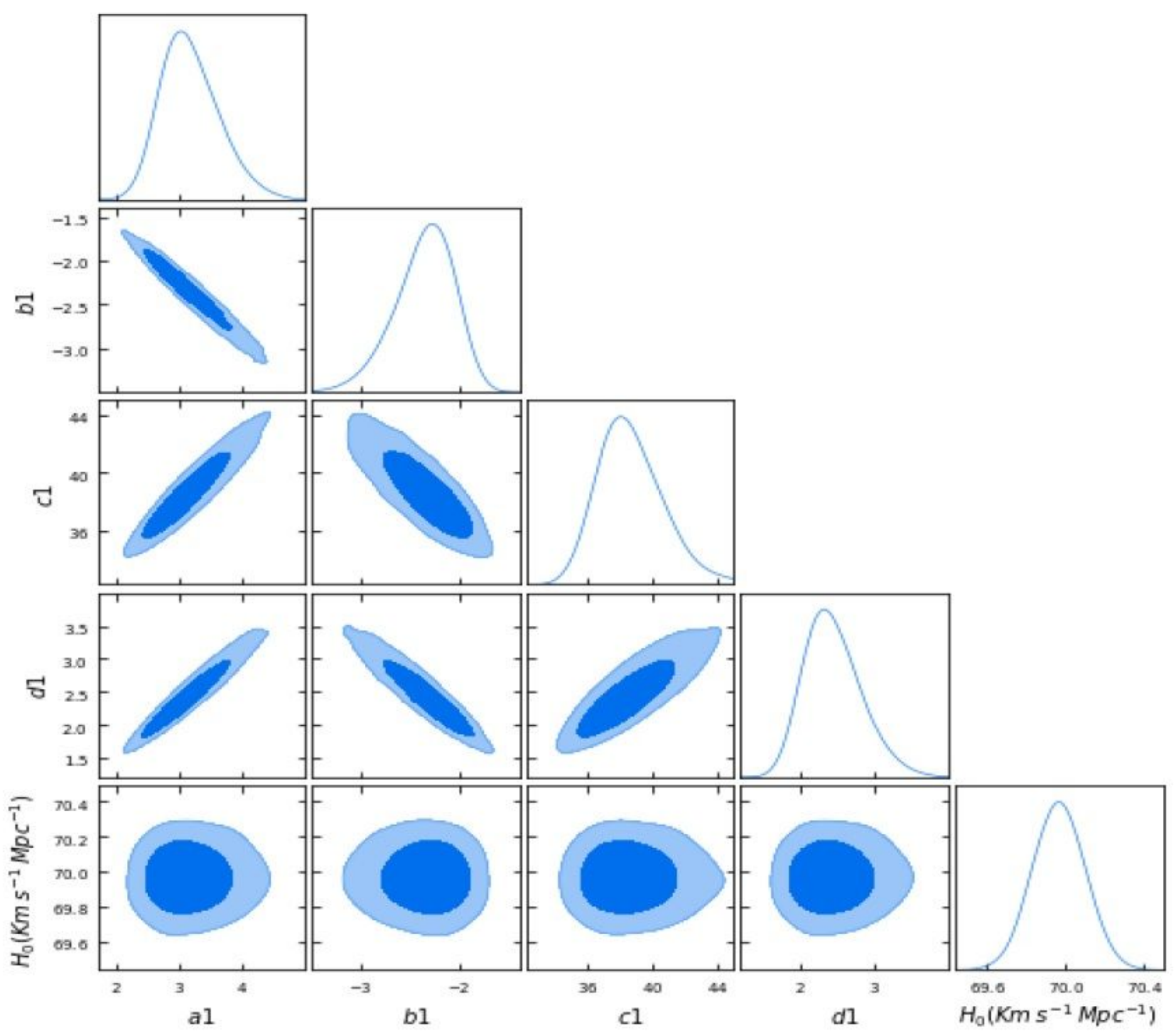

Figure 2

Result of the cosmological fit for $\mathrm{HO}$ considering both GRBs and SNe la data and by fixing $\Omega \mathrm{M}=0.3$ at a fiducial value and $w=-1$. 

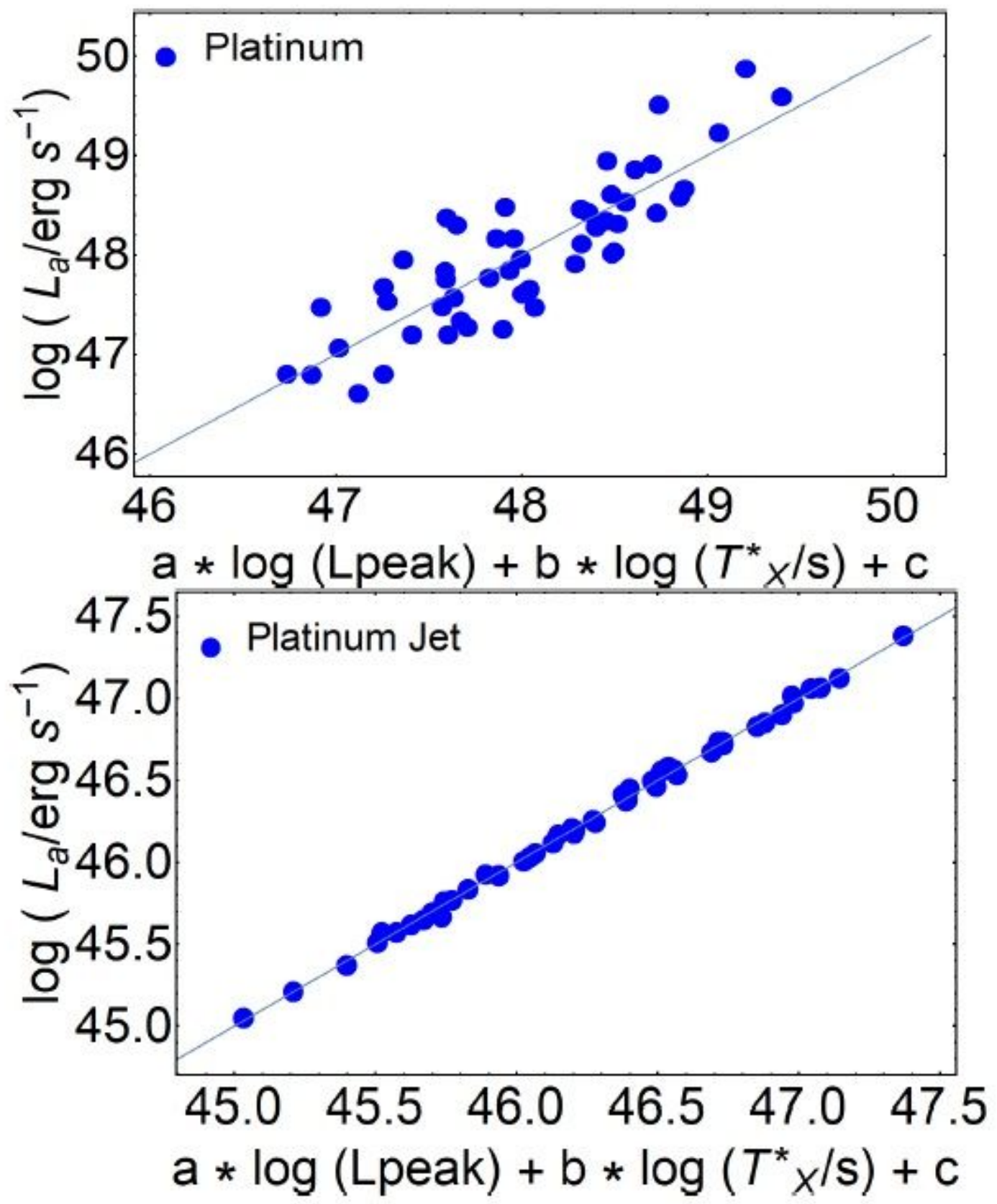

Figure 3

Upper panel: The isotropic fundamental plane relation in the isotropic case projected in a 2D plot. Lower panel: the jetted fundamental plane corrected for the jet opening angle again projected in 2D. 


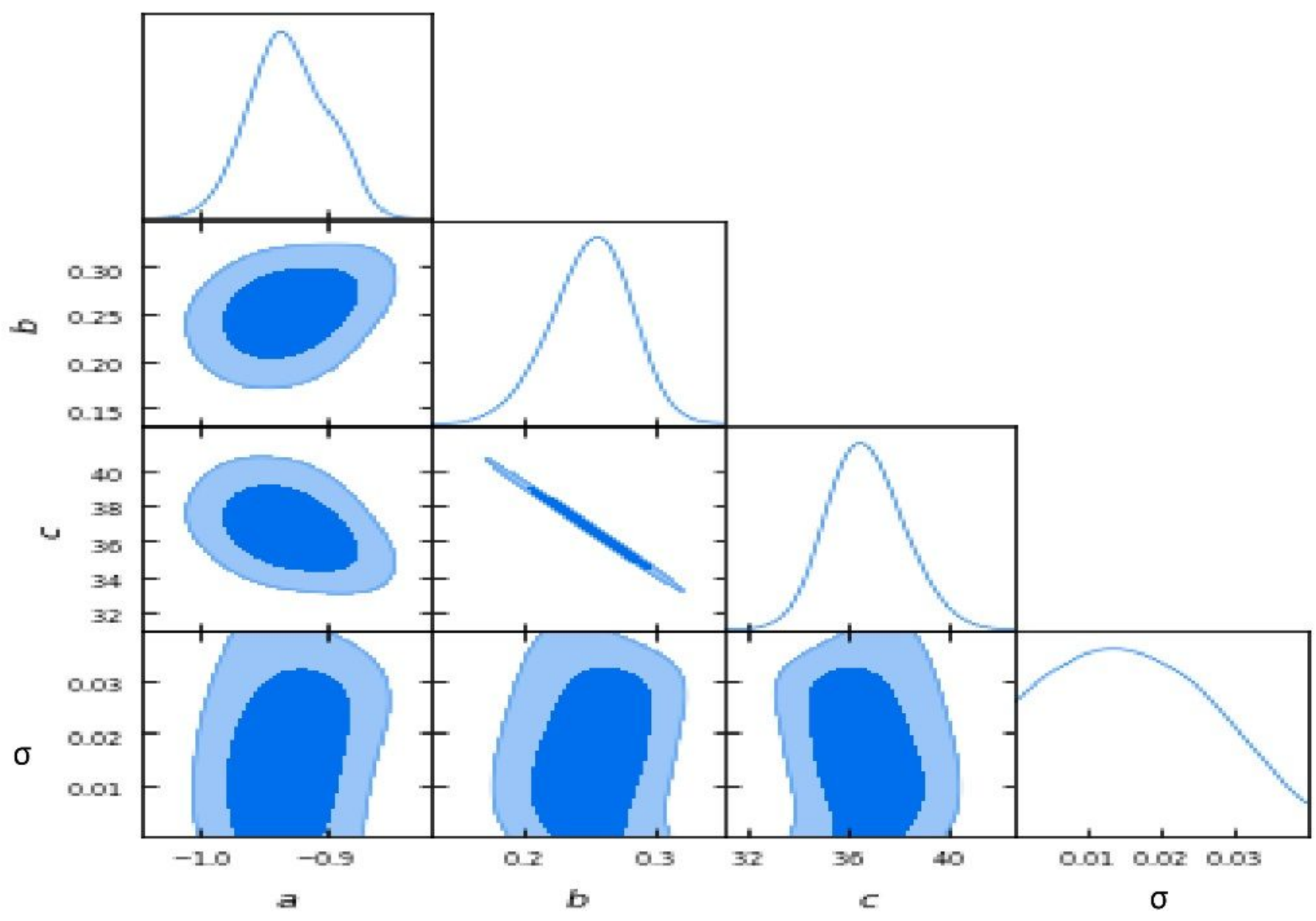

Figure 4

Contour plots at $68 \%$ and $95 \%$ level (the darker blue and the lighter blue, respectively) for the best fit parameters of the jetted fundamental plane of the platinum sample. 


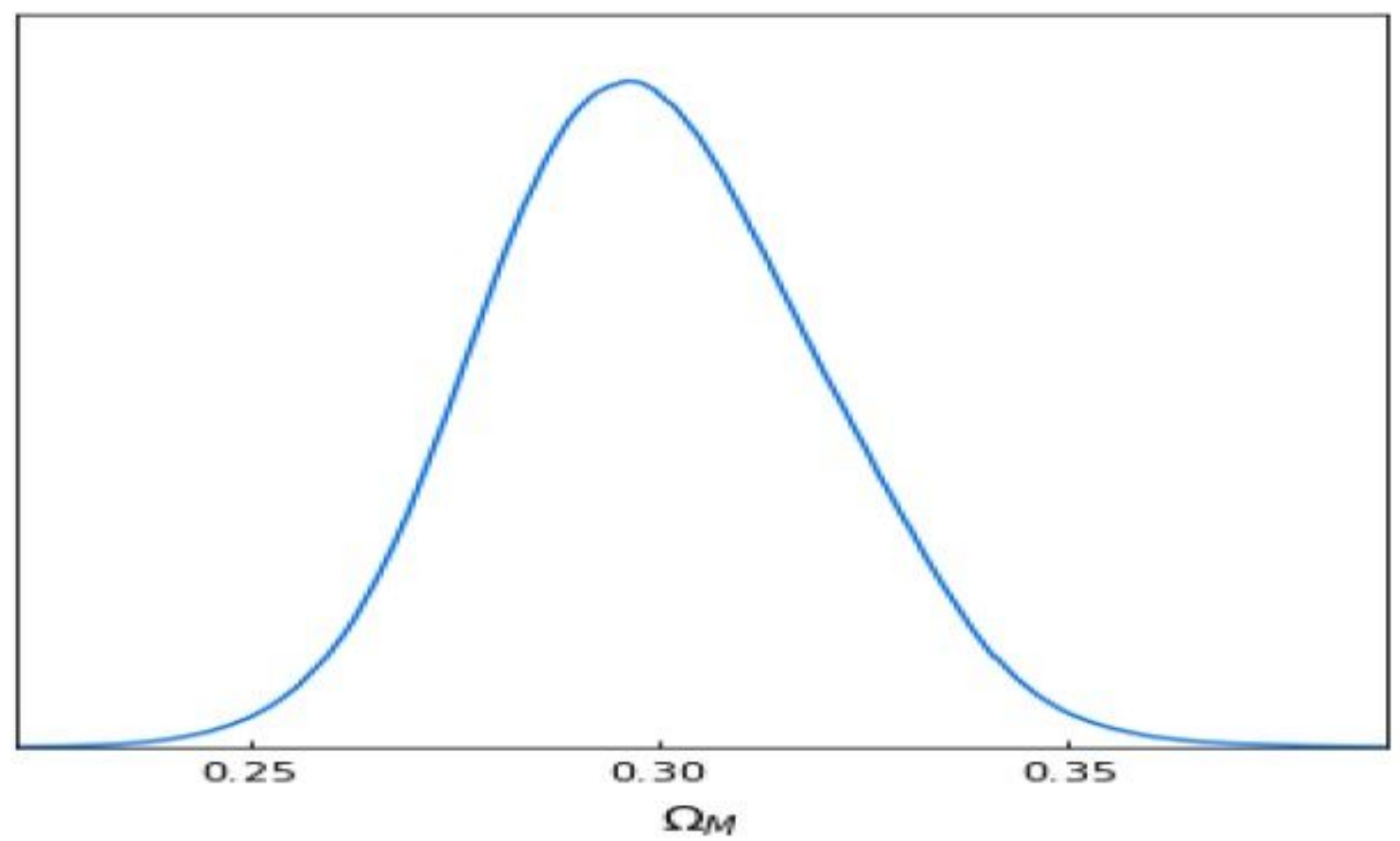

Figure 5

Result of the cosmological fit for $\Omega \mathrm{M}$ considering only SNe la data and fixing $\mathrm{H} 0=70 \mathrm{Km} \mathrm{s}-1 \mathrm{Mpc}-1$ and $\mathrm{w}=-1$ at fiducial values.

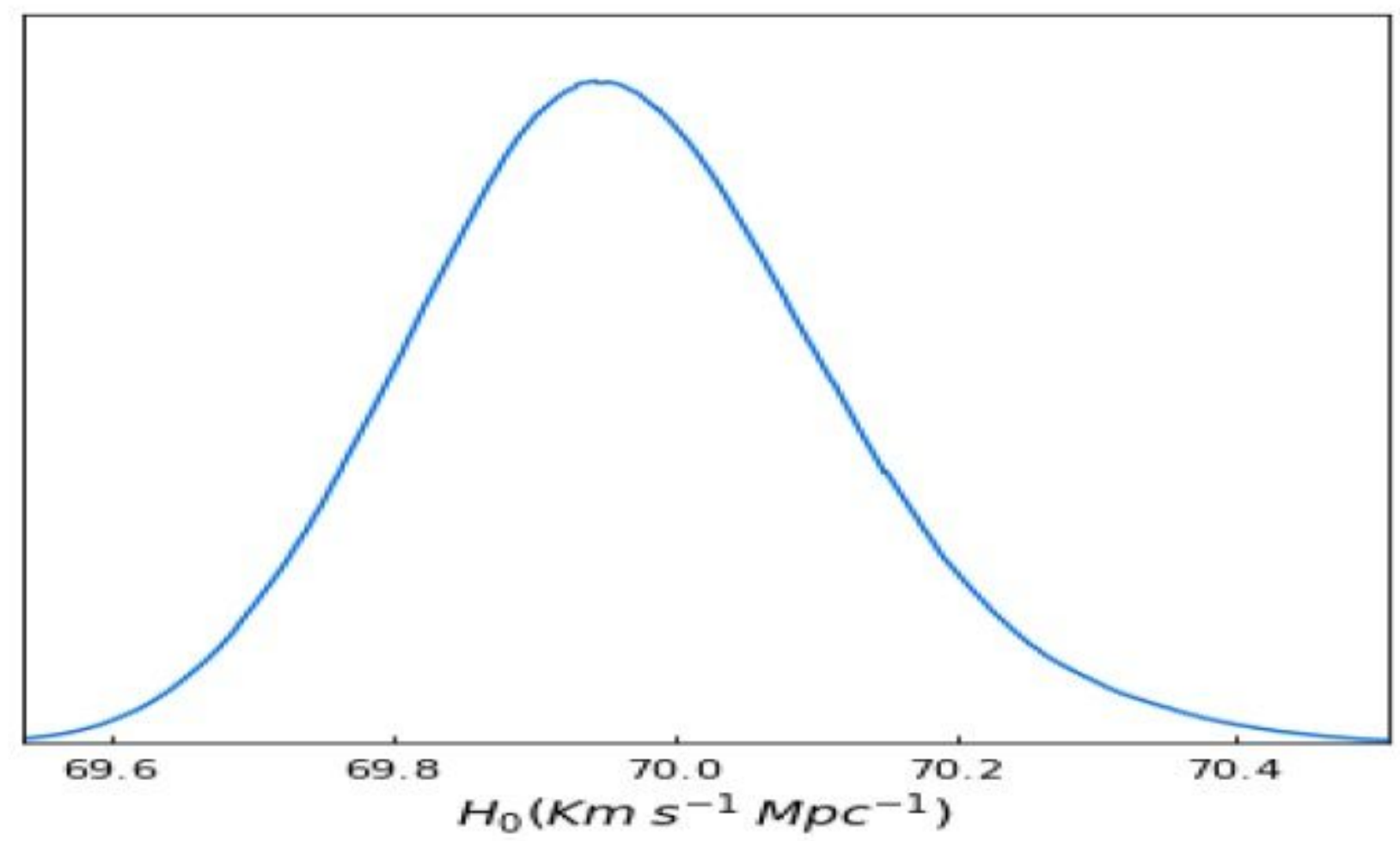

Figure 6 
Result of the cosmological fit for $\mathrm{HO}$ considering only SNe la data by fixing $\Omega \mathrm{M}=0.3$ and $w=-1$ at fiducial values.

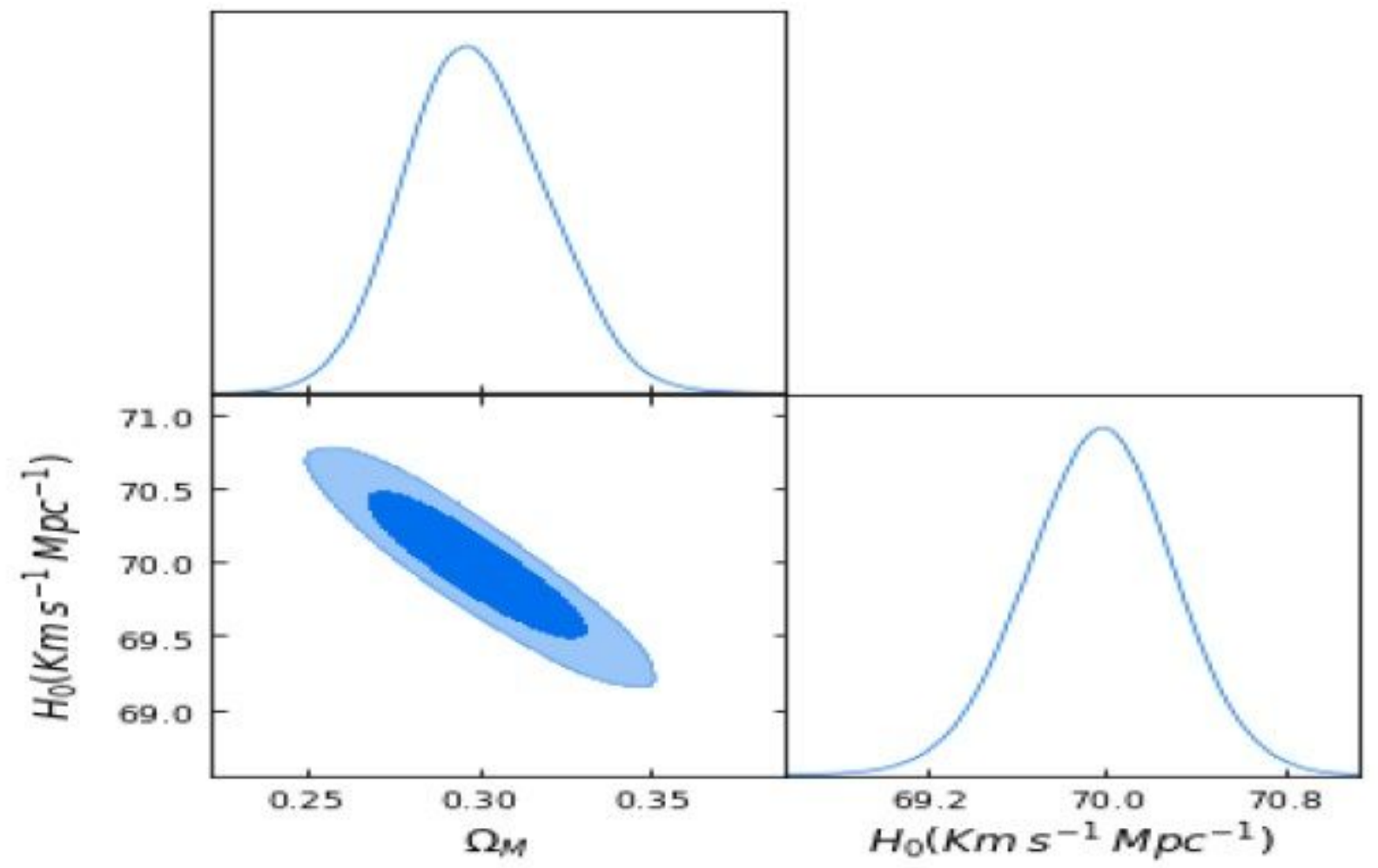

Figure 7

Contour plots of the parameters of only SNe la data at $68 \%$ (dark blue contours) and $95 \%$ confidence level (light blue contours) showing the result of the cosmological fit for $\Omega \mathrm{M}$ and $\mathrm{HO}$ fixing $\mathrm{w}=-1$.

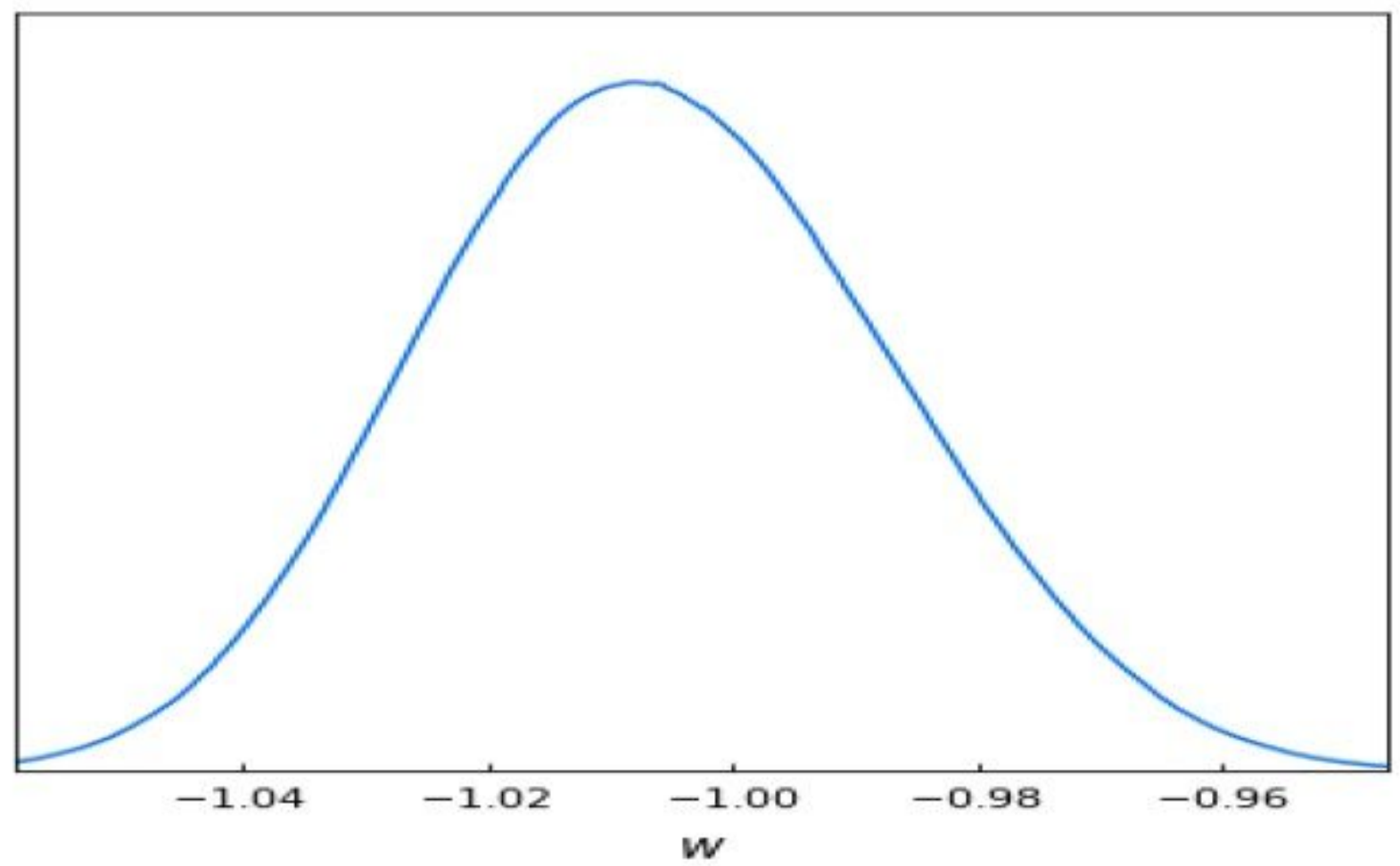


Figure 8

Result of the cosmological fit for w using only SNe la data and by fixing $\Omega M=0.3$ and $\mathrm{H} 0=70 \mathrm{Km} \mathrm{s}-1 \mathrm{Mpc}-$ 1 at fiducial values.

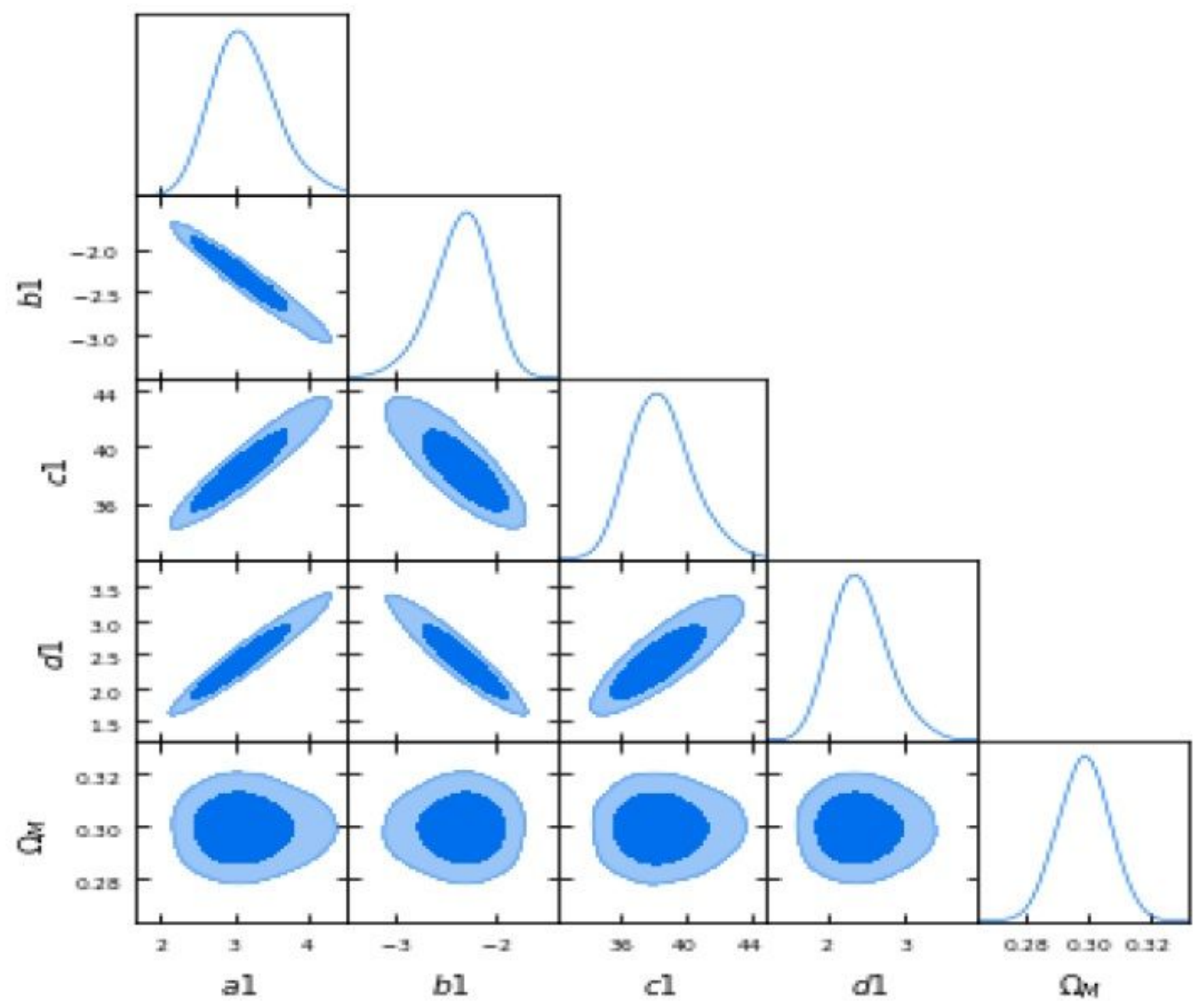

Figure 9

Result of the cosmological fit for $\Omega \mathrm{M}$ considering both $\mathrm{GRBs}$ and $\mathrm{SNe}$ la data and by fixing $\mathrm{H} 0=70 \mathrm{Km} \mathrm{s}-1$ Mpc-1 at fiducial value and $\mathrm{w}=-1$. 


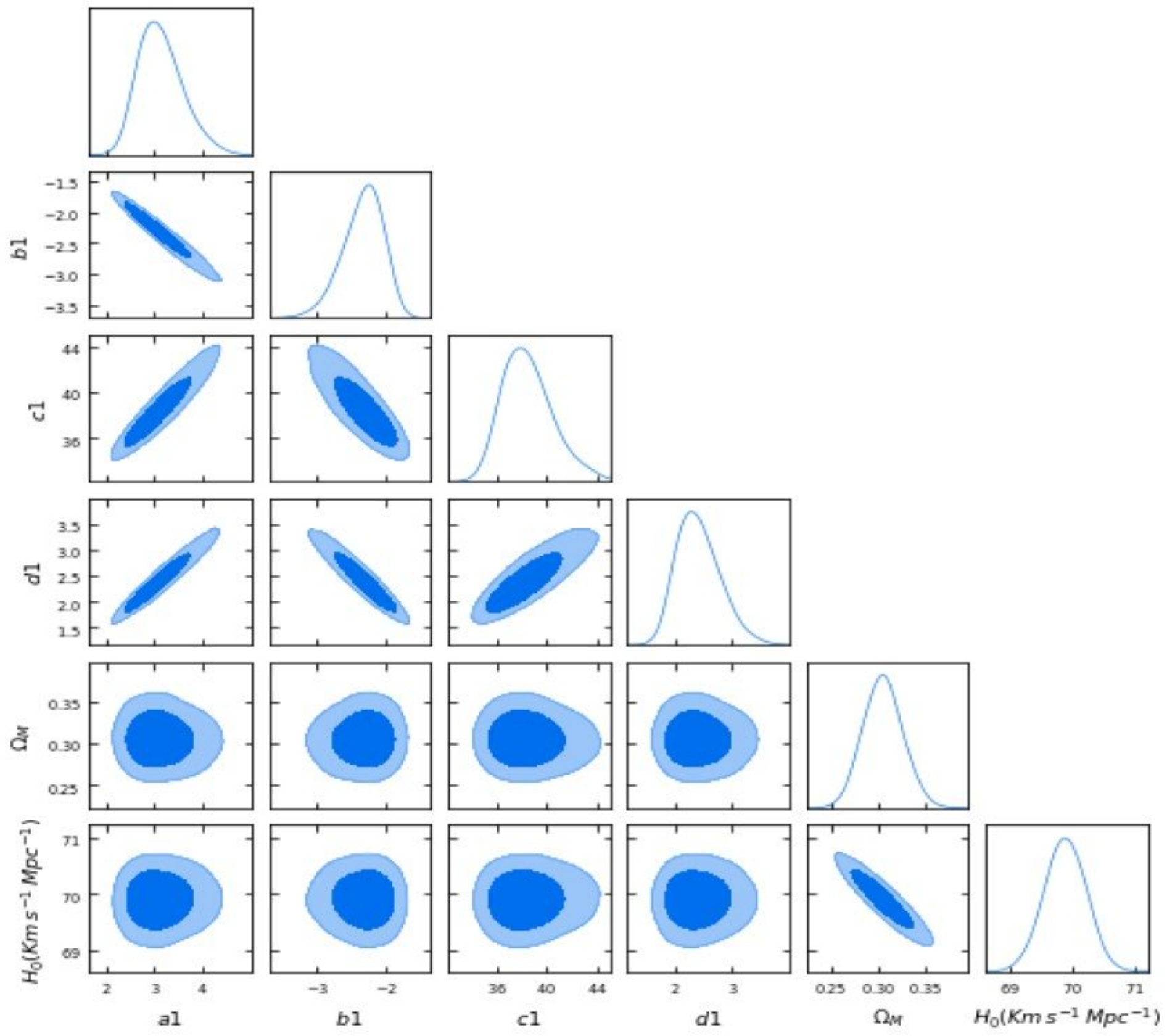

Figure 10

Contour plots of the parameters of the GRB platinum sample and SNe la together at $68 \%$ (dark blue contours) and $95 \%$ confidence level (light blue contours) and result of the cosmological fit for $\Omega \mathrm{M}$ and $\mathrm{HO}$ considering both GRBs and SNe la data. 


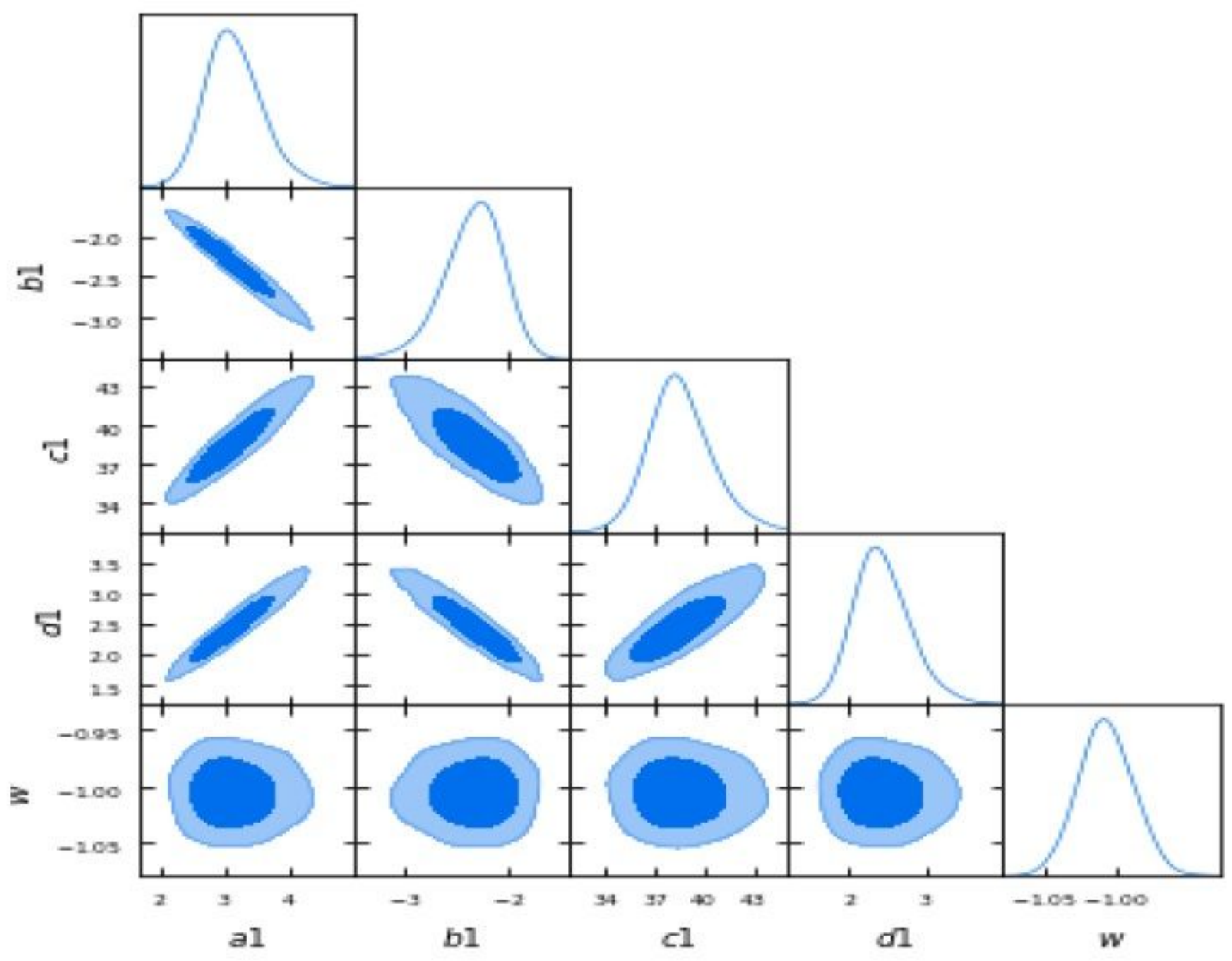

Figure 11

Contour plots of the parameters of the SNe la and the GRB platinum sample at $68 \%$ (dark blue contours) and $95 \%$ confidence level (light blue contours) and result of the cosmological fit for $w$ fixing $\Omega M=0.3$ and $\mathrm{HO}=70 \mathrm{Km} \mathrm{s}-1 \mathrm{Mpc}-1$ at fiducial values. 